\title{
Selenium/tellurium containing polymer materials in nanobiotechnology
}

\author{
Wei Cao, Lu Wang, Huaping $\mathrm{Xu}^{*}$ \\ Department of Chemistry, Tsinghua University, Beijing 100084, China. \\ Email: xuhuaping@mail.tsinghua.edu.cn
}

\section{Keywords}

Selenium-containing polymer; Tellurium-containing polymer; Self-assembly;

Nano-materials; Controlled delivery; Stimuli response.

\section{Summary}

Selenium/tellurium containing polymers have been attracting growing interest 
due to their unique properties and potential application as bio-nanomaterials. The introduction of selenium or tellurium chemistry into block copolymers endows self-assembled nanomaterials with novel stimuli responsiveness and enhanced sensitivity. This review article provides an overview on recent advances of selenium/tellurium containing polymers. We summarize the redox response and reversible self-assembly behaviors, $\gamma$-ray responsive systems for combined chemoand radio-therapies, and coordination-responsive systems for controlled delivery are highlighted. The review then reveals how selenium-containing polymers can modulate reactive oxygen species and work as gene delivery vehicles or self-delivery therapeutics.

\section{Introduction}

Selenium was discovered in 1817 by the Swedish scientist Jöns Jacob Berzelius, one of the founders of modern chemistry, who named it after selene, the Greek word of moon [1]. Located right below selenium in the chalcogen group of the period table is its congener tellurium, which was actually discovered 35 years earlier, in 1782 by Franz-Joseph Müller von Reichenstein. Tellurium was so named by Martin Heinrich Klaproth in 1798, after tellus, the Latin word for earth [2]. In the past few centuries, people have come to understand that, despite their strange odor, selenium and tellurium compounds do possess unique and interesting properties. For example, in 1970s selenium was proved to be incorporated into proteins to produce selenoproteins which perform as important antioxidant enzymes [3]. Great progresses has since been 
made to synthesize selenium-containing compounds with anti-oxidant properties ever since [4-7]. Similarly, after the pioneering use of tellurium compounds in organic synthesis, rising interest has been directed toward the field of tellurium chemistry [8-11].

Nanomaterials have been a thriving area of research over the past few decades. Block copolymers can self-assemble into a rich variety of complex nano-architectures with unique functions [12-14]. The incorporation of a diversity synthetic chemistry allows the fabrication of polymer materials with various stimuli responsiveness. One of the most promising applications is to work as controlled delivery vehicles [15-19]. Different stimuli have been integrated into delivery systems [19-25]. The recent trend, however, is to discover new materials that can alter their properties in response to changing environments with enhanced sensitivity so as to function in physiological conditions, since the biological signals or disease-related biomarkers are usually of ultra-low concentrations [26]. A feasible method to achieve this purpose is to introduce new functional moieties into polymer systems, which may broaden their applications and pushing forward the development of the current systems in the meantime.

Developing selenium- or tellurium- containing polymers with unique properties and potential application as bio-nanomaterials has been an interesting field of research which has been gathering attention and interest [27]. The unique chemistry of 
selenium or tellurium endows polymer materials with enhanced sensitivity and novel stimuli response properties. This review will provide an overview of the research related specifically to the selenium/tellurium-containing polymers as a new class of self-assembled nano-materials, but polyselenophenes and polytelluroohenes for optical and electronic applications are beyond the scope of this review. To begin with, we systematically summarize the recent progresses in the exploitation of the redox responses of the selenium-containing polymer nano-assemblies. Next, dynamic reversible self-assemble systems utilizing selenium chemistry are discussed, including switchable catalytic bioactivity at different states of oxidation. We then review the innovative $\gamma$-ray responsive delivery systems for their potential to combine chemoand radio- therapies, and further demonstrate how tellurium could increase the sensitivity of $\gamma$-ray response. Specifically, strategies for fabricating competitive-ligand regulated coordination responsive systems and gene delivery vectors are summarized. Furthermore, we highlight the selenium-containing polymer materials with novel functions such as the catalytic elimination of reactive oxygen species (ROS), or the self-delivery of therapeutics, featuring the biological activity of selenium groups. Finally, the future outlook for perspectives and challenges are discussed at the end of the paper. 


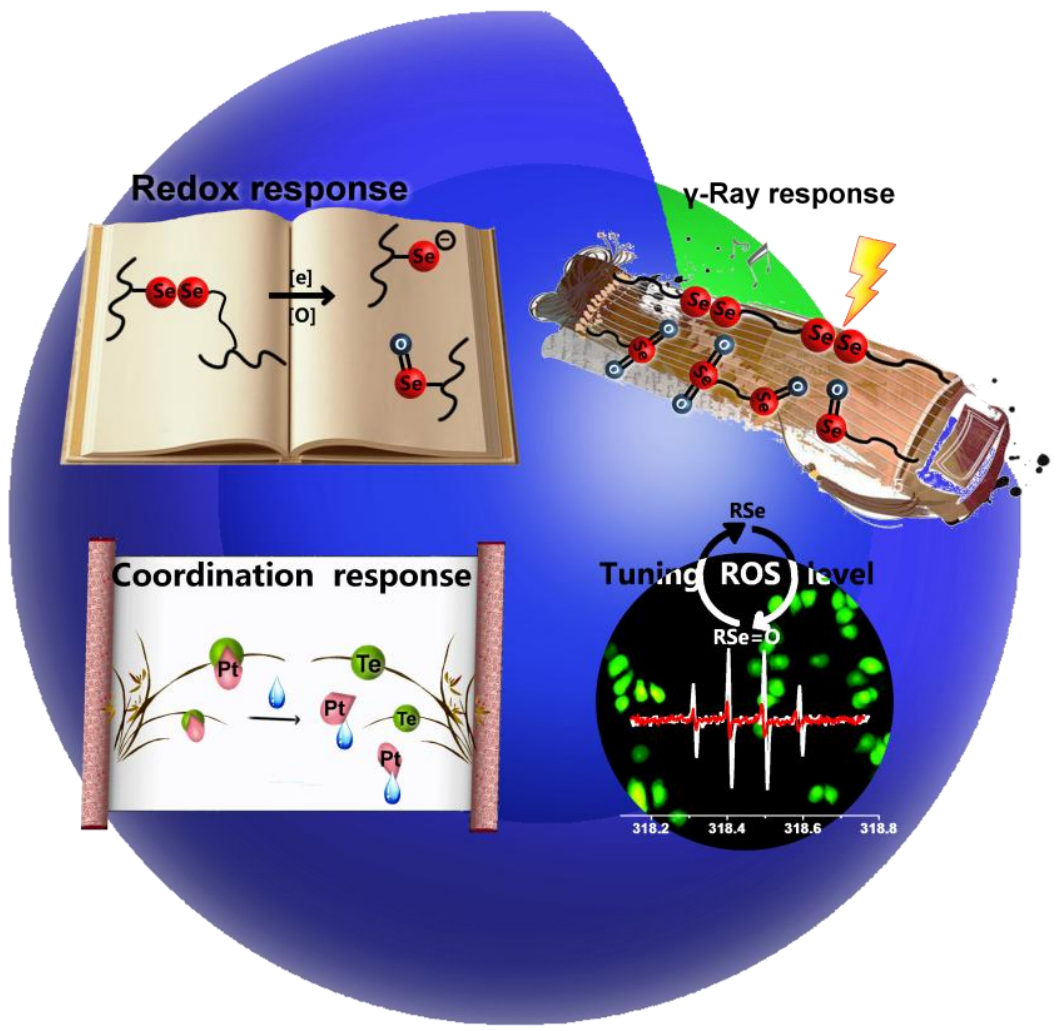

Scheme 1. The selenium/tellurium containing polymers and their representative applications in nanobiotechnology.

\section{Redox responsive materials}

Redox reactions, or those involving the transfer of electrons, are ubiquitous in our daily life, such as combustion, photosynthesis, batteries, etc. Redox reactions take part in processes that are indispensable to all life forms, such as photosynthesis or aerobic respiration. On a higher level, various pathways of signal transduction rely on the generation and transportation of electrons. Imbalanced redox conditions are known to cause various disorders in cells or organs [28].

One of the most charming characters of selenium-containing polymers is their redox response [27]. Selenium-containing compounds are blessed with unique redox 
properties. It still remains a challenge to fabricate a dual-redox responsive system by simple design. In this perspective, diselenide species are of interest as they are active to either oxidation as well as reduction. Previous studies on selenium-containing polymers are limited by its poor solubility in solvent and bad stability against oxidation. In 2010, our group succeeded in synthesizing a diselenide-containing block copolymer. Typically, selenium was introduced by the reaction of sodium selenide or sodium diselenide with an alkyl bromide. The sodium selenide or sodium diselenide was prepared from the material of selenium powder and sodium borohydride and used immediately after preparation. The main chain selenium-containing polymer is normally prepared by the stepwise polymerization of a selenium containing diol with slightly excess amount of Toluene-2,4-diisocyanate (TDI). Polyethylene glycol (PEG) is attached to the NCO groups on both ends to increase the solubility in water, thus making the polymer amphiphilic. The diselenide or monoselenide groups on the hydrophobic polyurethane backbone can be protected by the microenvironment provided by the hydrophilic-hydrophobic-hydrophilic structures of block copolymers in aqueous environment.

The diselenide-containing block copolymer demonstrated unique dual redox responsive behaviors (Fig. 1) [29]. Due to the hydrophilic PEG shell, the polymer self-assembled into stable spherical micelles with average diameter of about $76 \mathrm{~nm}$. They could load cargoes like Doxorubicin (Dox), Rhodamine B (RB) efficiently similar as typical polymer micelles. In the presence of redox stimuli, they could afford 
quick release in a controlled manner. It should be noted that the redox response could be triggered under very mild conditions. The loaded cargo was totally released within $5 \mathrm{~h}$ after the addition of $0.01 \% \mathrm{H}_{2} \mathrm{O}_{2}$, while upon treatment with $0.01 \mathrm{mg} / \mathrm{mL}$ glutathione $(\mathrm{GSH})$, the cargo was also released within $5 \mathrm{~h}$. The dual response of the selenium-containing polymer micelles is attributed to the redox activity of Se-Se bonds: it could be cleaved either by oxidation to seleninic acid or reduction to selenol in different redox environments. As cellular or organism disorders are often related to intricate redox changes in physiological environments, the diselenide containing polymers may serve as promising candidates for future development of programmable responsive biomaterials.

a)
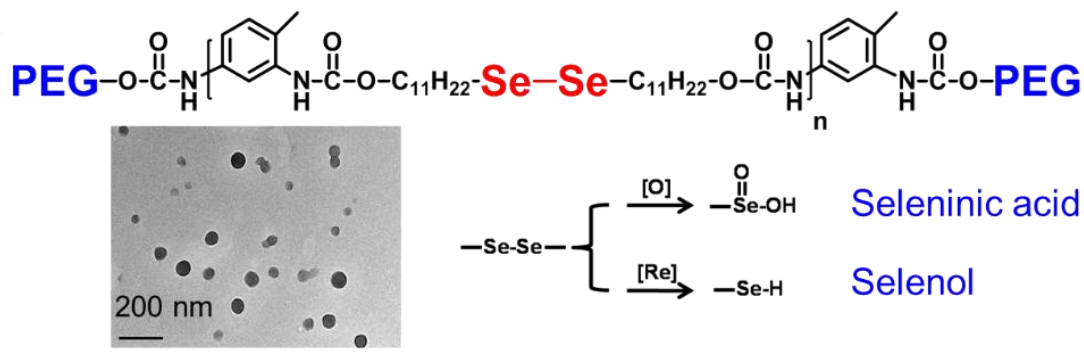

b)
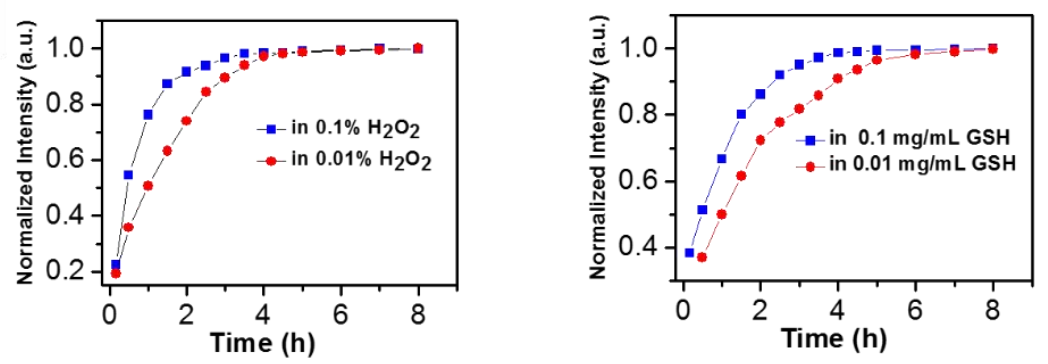

Fig. 1 Dual redox responsive diselenide-containing block copolymers. The PEG-PUSeSe-PEG micelles could be broken by either oxidants or reductants of very low concentrations. Fig $\mathrm{b}$ shows the release behaviors triggered by $0.01 \%, 0.1 \%$ $\mathrm{H}_{2} \mathrm{O}_{2}$ or $0.1 \mathrm{mg} / \mathrm{mL}, 0.01 \mathrm{mg} / \mathrm{mL}$ GSH. Copyright 2009 American Chemical Society 
[29].

After recognizing the redox responsiveness of the diselenide-containing polymer, a coassembly study shed light on how to achieve more complex diselenide-containing systems via supramolecular approach. Our group reported a general approach to fabricate stimuli responsive system by the coassembly of diselenide-containing block copolymers and polymer lipids (Fig. 2) [30]. Coassembly can integrate the merits of both building blocks, that is, biocompatibility of polymer lipids and dual-redox responsiveness of diselenide-containing block copolymers. By adding a little amount of dual redox responsive polymer into the polymer lipid system (mass ratio between polymer lipid and diselenide block copolymer is 10:1), the coassemblies retained good redox responsiveness. Thus, in the presence of $0.1 \% \mathrm{H}_{2} \mathrm{O}_{2}$ or $0.05 \mathrm{mM} \mathrm{GSH}$, most of the coassemblies were disrupted. Good responsiveness could be achieved even though only a little amount of diselenide-containing block copolymer was involved. Owing to the good biocompatibility of polymer lipids, it is suitable for further application under physiological conditions. Together with the good biocompatibility owing to the polymer lipids, further applications of this coassembly system in physiological conditions could be envisaged. 


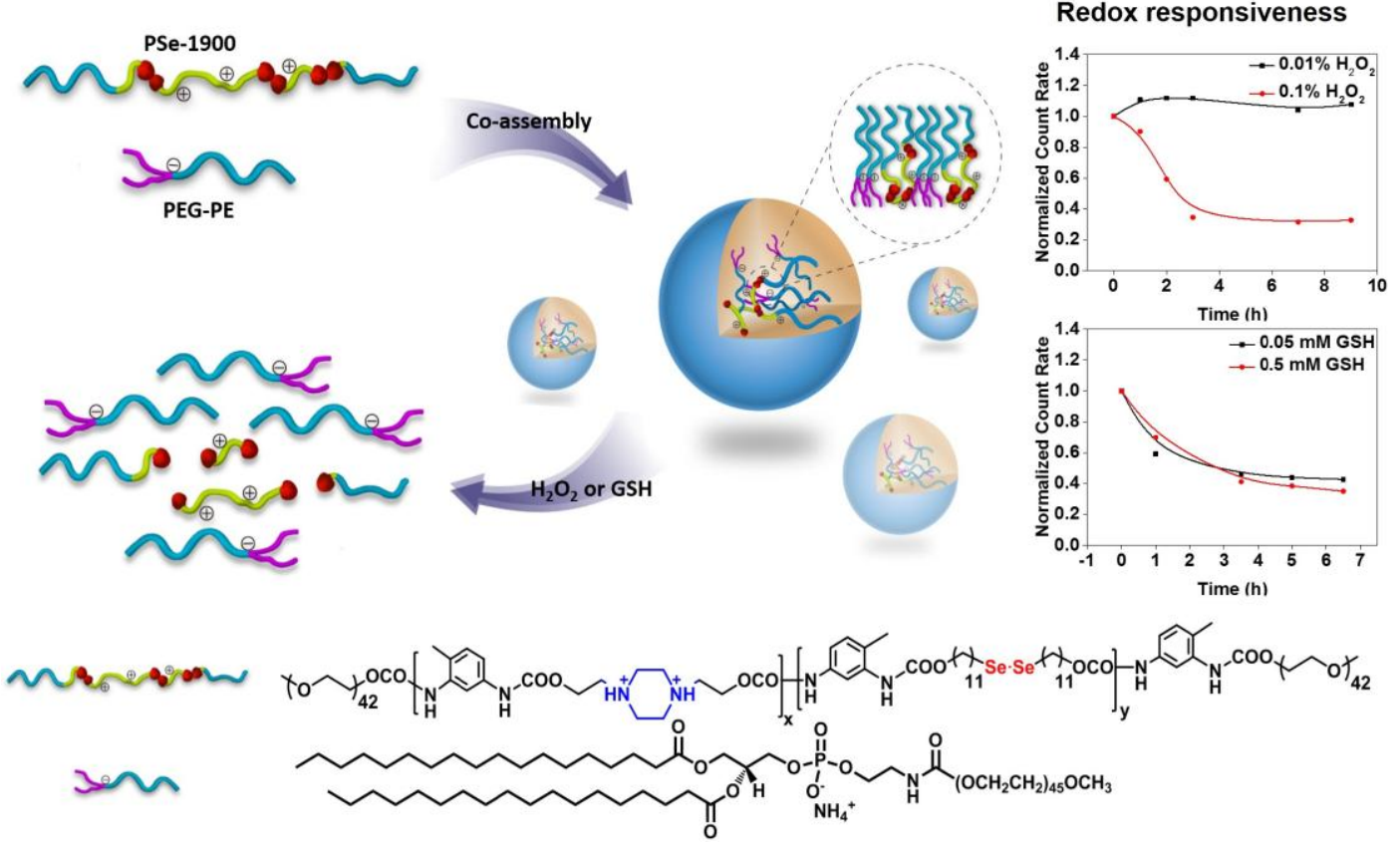

Fig. 2 Dual redox responsive coassemblies of diselenide-containing block copolymers and polymer lipids: the right of the figure is the normalized count rate of the coassemblies in the presence of $\mathrm{H}_{2} \mathrm{O}_{2}$ or GSH stimuli. Copyright 2014 American Chemical Society [30].

After the discovery of the dual redox responsiveness of the diselenide bond, we wondered whether diselenide-containing materials could also respond to light since the diselenide bond is relatively weak. Red light is safe and can penetrate deep in tissue. We reported a red-light responsive release of anticancer drug Dox from PEG-PUSeSe-PEG micelles with porphyrin as a mediator (Fig. 3) [31]. Singlet oxygen was produced by porphyrin when irradiated by red light and subsequently oxidized the diselenide bonds, which resulted in the disassembly of the micelles and the release of the loaded Dox. As thicker PEG shell could inhibit the contact and reaction of the singlet oxygen with diselenide bonds, the diselenide containing 
micelles also showed PEG modulated light-responsive behaviors. It was demonstrated that this line of diselenide-containing polymers were biocompatible and showed no influence on the proliferation of L-02 cells in $24 \mathrm{~h}$ with different PEG lengths.
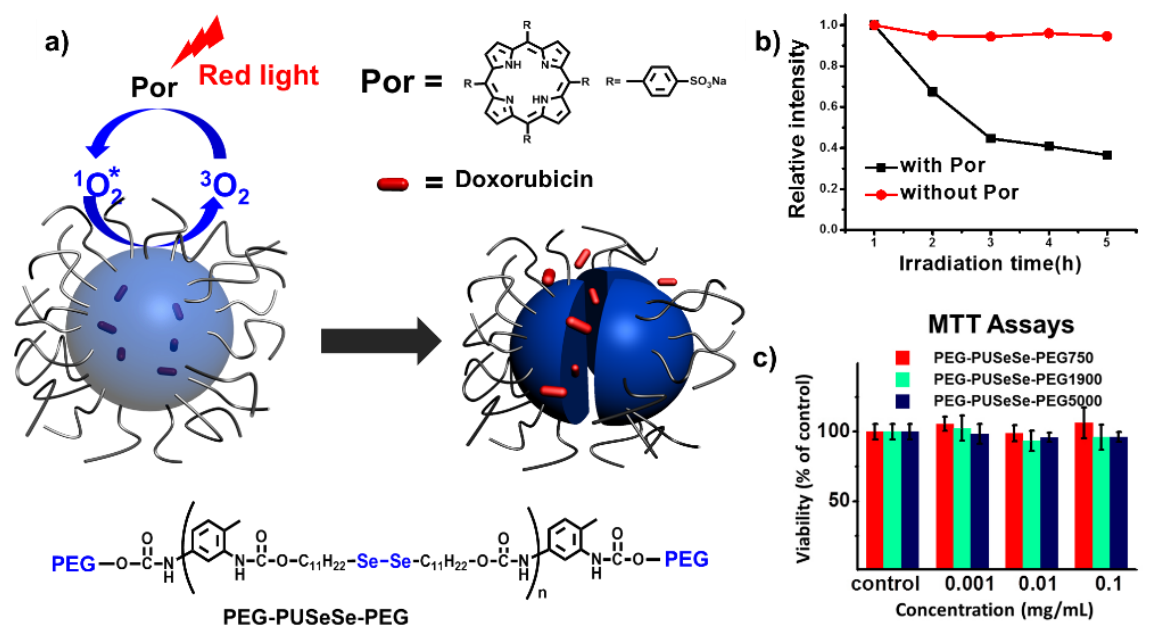

Fig. 3 Red-light responsive diselenide-containing polymer micelles. When radiated by red light in the presence of porphyrin, the polymer micelles could be partially broken and release the loaded Dox (Fig b). MTT assays showed the good compatibility of polymers with different molecular weight. Copyright 2013 Royal Society of Chemistry [31].

While the light responsive efficiency is still not satisfactory in the above system, we further showed structural engineering could be utilized to enhance the efficiency of light induced disruption of self-assembled structures. A positively-charged diselenide-containing polymer was designed and synthesized, and then alternatively deposited with the photosensitizer to place the ROS-generating photosensitizer and the active diselenide bonds in close vicinity to each other (Fig. 4) [32]. The film 
obtained could be gradually degraded under the irradiation of mild white light. It is demonstrated that when incorporated into the film, porphyrin shows a 100-fold increase in the efficiency of singlet oxygen production, compared to that in a simple solution. Furthermore cells could grow regularly with polygonal morphology on the films and almost covered the entire slide after $72 \mathrm{~h}$. As the light used in this work is even weaker than the sunlight, it is anticipated that this work may further be developed into a platform technology to combine chemotherapy with photodynamic therapy.

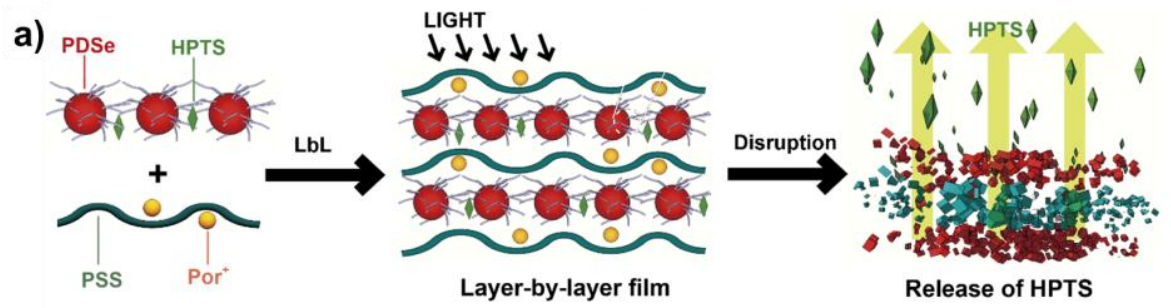

b)
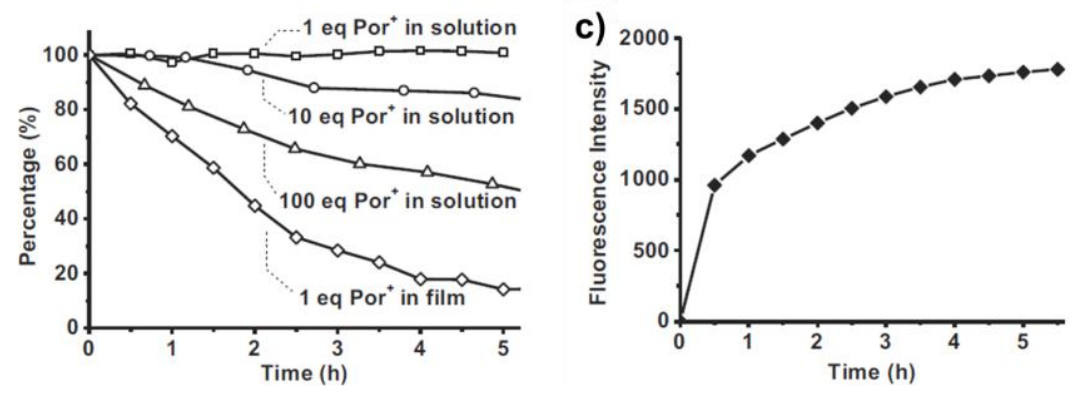

Fig. 4 White-light responsive selenium-containing multilayer films based on Layer by Layer (LbL) technique. The photosensitizer was loaded in the film by LbL methods and the efficiency of the singlet-oxygen increased significantly compared with photosensitizer in solution (Fig b). White-light triggered release of model compound form the film could as be observed (Fig c). Reproduced with the permission of 2013 Wiley Publishers [32]. 
The sensitive response of the above systems may be the additive effect of two factors: one is the active nature of the selenium chemistry, and the other is the amplification effects of the large number of repeating diselenide units, since each cleavage of the Se-Se bond leads to the degradation of the polymer backbone. It is also interesting to know whether it is possible to fabricate sensitive responsive system based on amphiphilic polymers with only one single diselenide bond in the polymer backbone. Jin and coworkers reported an example of polymers with one single diselenide bond on the main chain, PEG-PPG-Se-Se-PPG-PEG and PEG-Se-Se-PPG [33, 34]. Self-assembled structures from these polymers were also sensitive to oxidation. $0.5 \% \mathrm{H}_{2} \mathrm{O}_{2}$ could trigger the morphology change and the release of the loaded molecules. So we believe that the sensitive response of selenium-containing polymers is largely attributable to the active nature of the diselenide bond itself.

Apart from changing the number of diselenide bonds, it is also of vital importance to control their inherent redox activity. Zhang and coworkers demonstrated a supramolecular strategy to modulate the reactivity of Se-Se bonds [35]. Selenocystamine (SeCy) by itself was rather sensitive to redox stimuli, but upon binding with a host molecule to cucurbit[6]uril $(\mathrm{CB}[6])\left(\mathrm{K}_{\mathrm{a}}=5.5 \times 10^{6} \mathrm{M}^{-1}\right)$ via host-guest chemistry, however, the Se-Se became inert. Further studies revealed that SeCy-CB[6] complex was $5 \times 10^{3}$ time less active toward oxidation than the free SeCy. Additionally, complete recovery of the redox properties could be achieved by 
disrupting the host-guest pair using a competitive guest $\mathrm{CB}[6]$. This supramolecular control over the reactivity provides a possible way to modulate the selenium redox process by changing the microenvironments.

While the diselenide bonds show advantages in redox response, the chemistry of selenium chemistry also indicates that the monoselenide compound can be oxidized to selenoxide or selenone. Unlike the diselenide bonds, no bond cleavage occurs in the monoselenide oxidation. However, the amphiphilicity change between the oxidized state and the reduced state could still allow the possible design of stimuli-responsive nanomaterials. Our group reported the synthesis of monoselenide-containing polymer PEG-PUSe-PEG by similar stepwise polymerization methods [36]. Analogous to its diselenide-containing counterpart, the polymer PEG-PUSe-PEG could self-assemble to form spherical micelles and load the cargo Dox. The polymer micelles could be oxidized by $0.1 \% \mathrm{H}_{2} \mathrm{O}_{2}$ and release the Dox in a controlled manner. As a control, monosulfide-containing polymer was also fabricated and tested. However, both its oxidation percentage and the Dox release amount were much lower than the PEG-PUSe-PEG micelles, further confirming the sensitivity of selenium to oxidation (Fig. 5). Besides, our group also proposed a supramolecular strategy to fabricate mono-selenide containing polymers [37]. Through the electrostatic interaction driven complex between PEG-b-PAA and a selenide surfactant Phenylselenide-1-undecyl triethylamonium bromide, an oxidation-sensitive supra-amphiphile could be formed. By changing the ratio between the two species, the self-assembly and controlled 
release behaviors could be tuned, which could facilitate the application of selenide-containing nanocontainers under mild circumstances.

a)
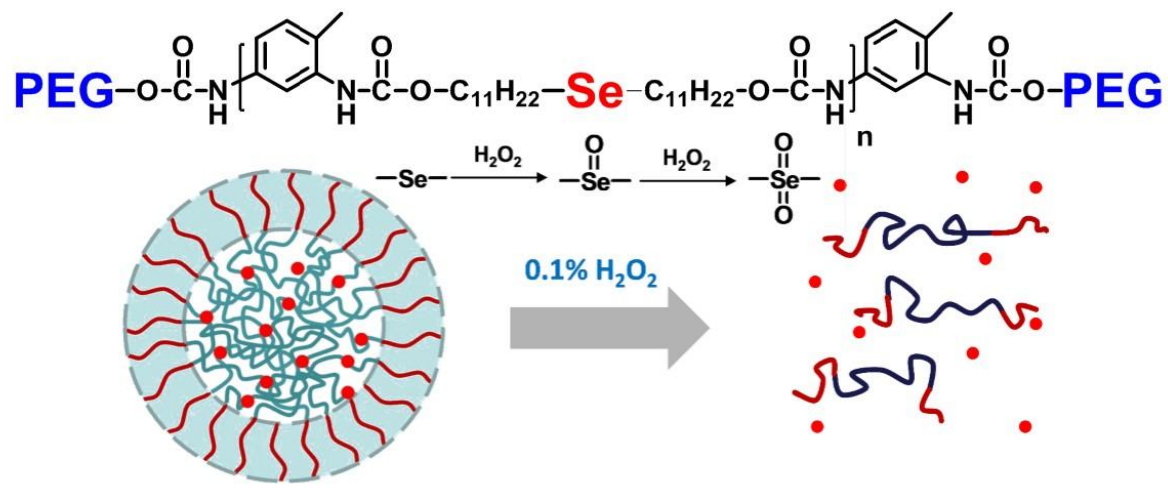

: Doxorubicin

b)

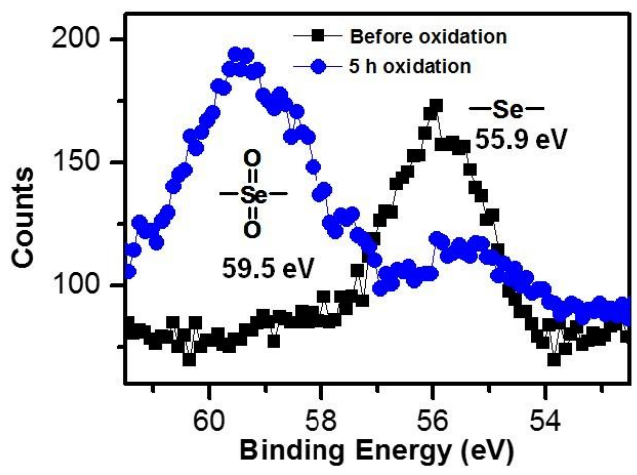

c)

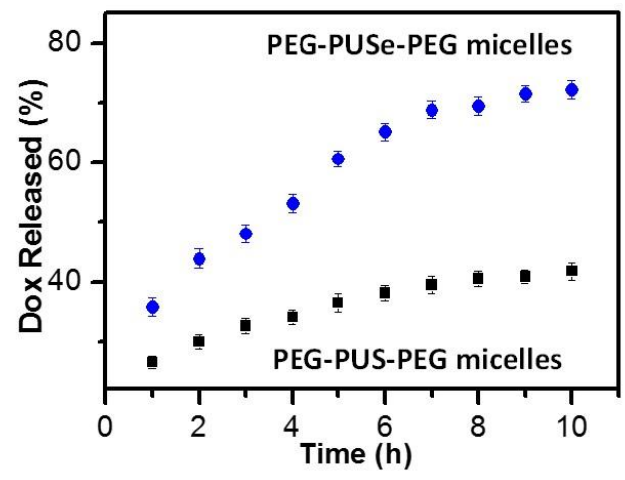

Fig. 5 The monoselenide containing polymer and its oxidation-responsive behaviors. b) XPS spectra of the PEG-PUSe-PEG sample before and after oxidation. c) oxidation-triggered release of Dox from the monoselenide containing polymer micelles and the monosulfide control. Copyright 2010 Royal Society of Chemistry [36].

The development of nano-materials also depends on the discovery of new chemistries. The periodic table is a chart that shows how elements are related to each 
other. Selenium is known for its rich redox properties. As tellurium sits near the bottom of the chalcogens family, the electronegativity of tellurium is lower than that of selenium [38]. As a result, the tellurium compounds are expected to possess higher sensitivity to ROS [39-42]. Our group introduced tellurium into the backbone of amphiphilic block copolymers and found that the nano-size self-assemblies in aqueous media possessed with ultra-sensitive response to ROS (Fig. 6) [43]. According to the cyclic voltammetry measurement of selenide and telluride compounds, the oxidative potential of telluride compounds are much lower than the selenide counterparts. In the presence of $100 \mu \mathrm{M} \mathrm{H}_{2} \mathrm{O}_{2}$, the tellurium-containing polymer micelles could transform into a series of different morphologies from swelled state to irregular aggregates. The $\mathrm{H}_{2} \mathrm{O}_{2}$ concentration employed in this line of research is $0.0003 \%$ in volume fraction, which is the typical biologically relevant concentration [44]. 
a)
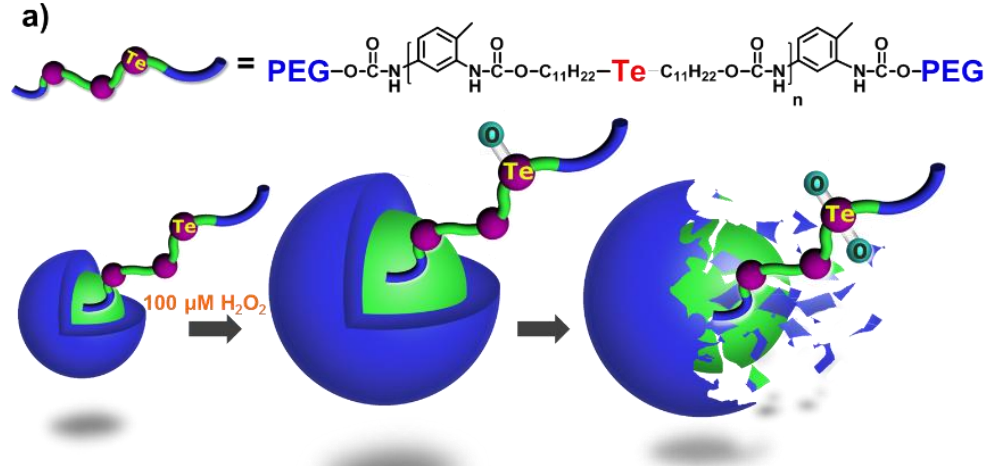

b)

c) $\mathrm{HOOCC}_{10} \mathrm{H}_{20}-\mathrm{Te}-\mathrm{C}_{10} \mathrm{H}_{20} \mathrm{COOH}$

Introducing tellurium into polymers is also a general way to endow a system with ROS-sensitivity. We synthesized a water-soluble tellurium-containing hyperbranched polymer via $\mathrm{A} 2+\mathrm{B} 3$ polymerization followed by $\mathrm{PEG}$ termination and demonstrated that it could form ROS-responsive aggregates (Fig. 7) [45]. The hyperbranched polymer micelles swelled a lot under biologically relevant concentration of $\mathrm{H}_{2} \mathrm{O}_{2}$, which could be expected to be applicable in the fields of ROS elimination and controlled release under mild conditions. The tellurium-containing polymer is a significant advance towards ultra-sensitive oxidation responsive 
materials.
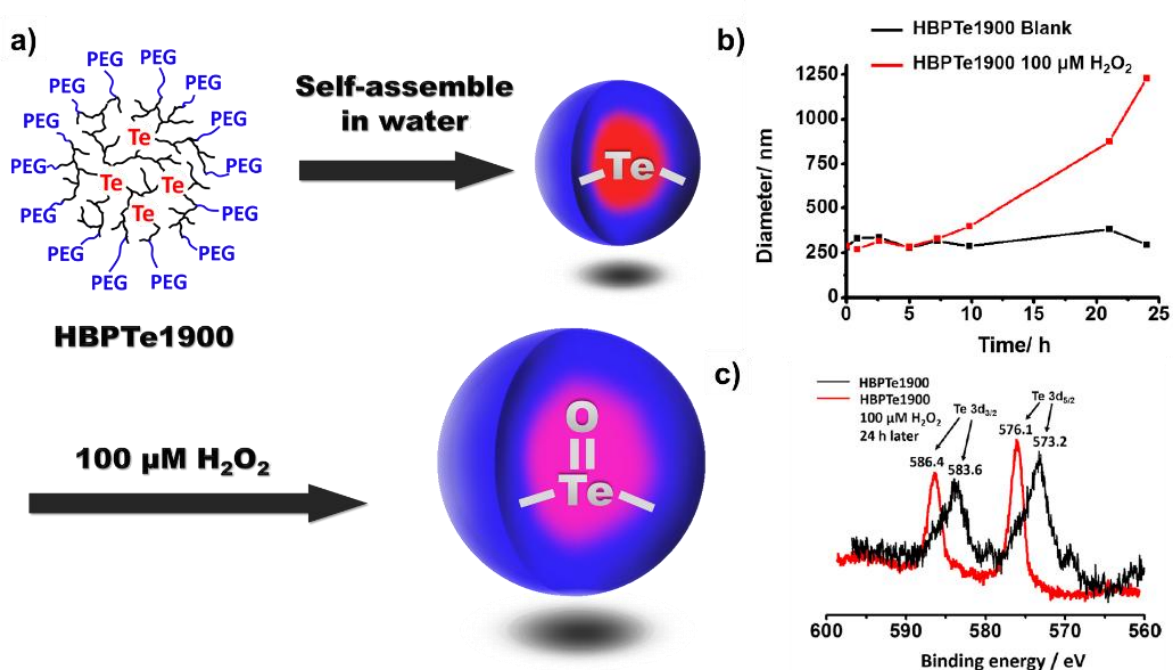

c)

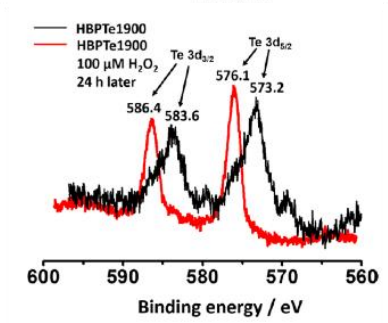

Fig. 7 A water-soluble hyperbranched polymer could form ROS-responsive aggregates. The micelles swelled a lot under the stimulus of $100 \mu \mathrm{M} \mathrm{H}_{2} \mathrm{O}_{2}$ (Fig b). The tellurium atoms were oxidized as indicated by the XPS analyzation in Fig c. Copyright 2015 Royal Society of Chemistry [45].

Stepwise polymerization is a powerful way to synthesize polymers with different moieties on the main chain, which makes it feasible to integrate various functions into polymers, and makes the polymers easy to degrade. However, the drawback of stepwise polymerization is poor control over polydispersity index (PDI). As gaining more control over the structure and PDI of polymers is an ultimate objective for polymer chemists, selenium-based mediators have been explored as a novel method in living free radical polymerization areas. Due to the lower bond energies of C-Se bond than C-S bond, the selenium containing agents are expected to have better control over radical polymerization. Zhu and coworkers studied selenium containing chain 
transfer agent (CTA) for reversible addition-fragmentation chain transfer (RAFT) polymerization of Vinyl Acetate (VAc). The selenium CTA showed clear living behaviors during the VAc polymerization and better control over its sulfur counterpart. However, the relatively large PDI seems to be a little far from satisfactory [46, 47]. Zhu and coworkers also utilized a series of selenocarbonates as mediators for the polymerization of styrene and methyl acrylate, which showed similar controllability to their sulfur analogues $[48,49]$.

\section{Dynamic and reversible self-assembly materials}

Dynamic behaviors are well controlled in nature: The constant and reversible polymerization and depolymerization of microtubules, the activation and deactivation of creatine phosphokinase, etc. The function of natural systems relies largely on the reversible systems. While some research on self-assembly pays attention to the static form, controlling the dynamic change of synthetic materials is of vital importance for the "bottom-up" fabrication of nanoscale materials [50, 51]. Selenium-containing polymers offer possibilities for dynamic functions under mild conditions.

Our group gained redox-responsive, reversible control over self-assembled nanostructures by introducing mono-selenide moiety to the side chains of selenium-containing polymers (Fig. 8) [52]. $\mathrm{PhCH}_{2} \mathrm{Se}\left(\mathrm{CH}_{2}\right)_{11} \mathrm{OH}$ was grafted to PEO- $b$-PAA to impart amphiphilicity. The PEO- $b$-PAA-Se self-assembled into spherical micelles with diameters of about $30-40 \mathrm{~nm}$, and transformed into irregular 
aggregates after oxidation by $0.1 \% \mathrm{H}_{2} \mathrm{O}_{2}$. The oxidation-triggered change could be reversed by adding reductive vitamin $\mathrm{C}(\mathrm{Vc})$. As revealed by DLS measurements, the reversible cycles could be repeated for at least 7 times. Detailed analysis by NMR and MS revealed that the chemistry behind the morphological change was the oxidation of selenide to selenoxide structure, and the opposite for the reduction process. The reversible selenium chemistry facilitates the construction of dynamic self-assembled architectures.

a)
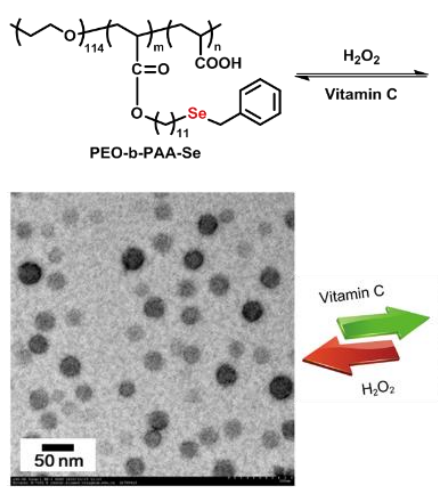

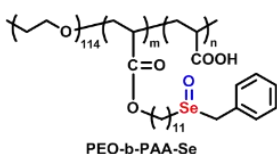

PEO-b-PAA-Se

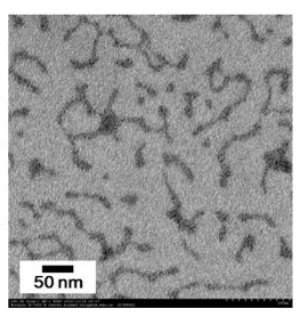

b)

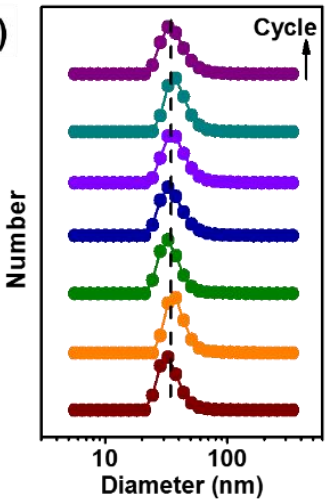

Fig. 8 Side-chain selenium-containing block copolymer and its reversible redox response. The polymer self-assembled into spherical micelles in reduced state and changed into irregular aggregates after oxidation to selenoxide structures (Fig a). The redox cycle could at least repeat for 7 times, as revealed by DLS in Fig b. Copyright 2011 Royal Society of Chemistry [52].

Redox systems are widely used to regulate biological activities, for example, The $\mathrm{NADH} / \mathrm{NAD}^{+}$has an almost ubiquitous presence for electron transfer reactions and protein posttranslational modifications. Using mild redox switches to achieve 
functional control is an interesting nature-mimicking issue in biomimetics. Xu, Yang and coworkers reported a supramolecular hydrogel system of selenium-containing polypeptides with reversible redox-controllable self-assembly and catalytic activity (Fig. 9) [53]. Selenoxide attached with peptide could be slowly converted to selenide by 1 equivalent of Vc in PBS. Subsequently, the solution turned into a hydrogel after $6 \mathrm{~h}$. The oxidized state form of the peptide self-assembled to spherical micelles, while the reduced state into long nanofibres and 3D entangled network for hydrogelation. Histidine was attached to the peptide and reversible catalytic properties for the hydrolysis of acetal-4-nitrophenol could be realized by redox control. The microenvironments of the catalytic histidine groups are different in the two states of oxidation, leading to different rates of hydrolysis. The $K_{\text {cat }} / K_{M}$ value was 1.1 for the gel and increased to 10.5 after the oxidation by $\mathrm{H}_{2} \mathrm{O}_{2}$ to the micelle state. When $\mathrm{Vc}$ was added into the micelle solution, the hydrolysis ability decreased again. Moreover, the hydrogel is biocompatible and shows esterase-like activity within the smooth muscle cells. This study will lead to biomimetic systems with promising functions.
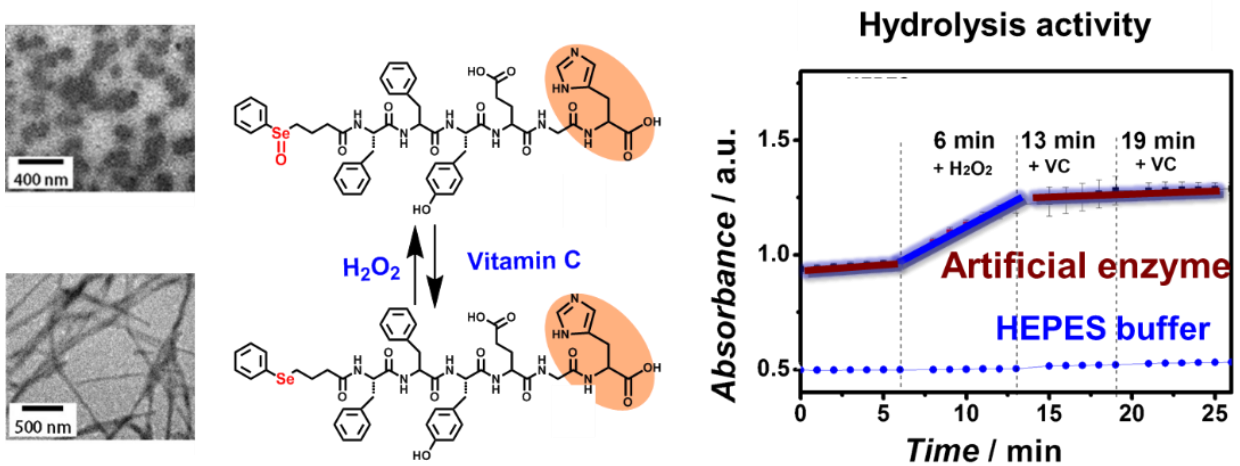

Fig. 9 Selenium-containing polypeptide with redox-controllable self-assembled 
properties and switchable catalytic activity. Mild redox condition change could trigger the transformation from micelles to fibers and the consequent sol-gel macroscopic change. The right figure shows the redox-switchable hydrolysis activity changes. Copyright 2013 WILEY-VCH [53].

Dynamic covalent bonds, which can be reversibly formed and cleaved in certain conditions, play significant roles in metabolic activities, such as the formation and cleavage of disulfide bonds in the protein folding process. Our group found a new kind of dynamic covalent bond Se-N, which can be formed between Se atom of a phenylselenyl halogen species (electron acceptor) and $\mathrm{N}$ atom of a pyridine derivative (electron donor) [54]. In the presence of an acid or base, the Se-N dynamic covalent bond can be reversibly formed or cleaved, respectively. Heating or using a strong electron donor (such as 4-dimethylaminopryidine, DMAP) can also dynamically cleave the Se-N bonds. This novel dynamic covalent bond could find new applications in fabrication of functional materials. Inspired by this work, $\mathrm{Xu}$, Wang and coworkers made use of Se-N interactions to tune the polymeric amphiphilicity of polystyrene-b-poly(4-vinylpyridine) (PS- $b$-P4VP) and realized one-step double emulsion (Fig. 10) [55]. After gradually adding phenylselenyl bromide ( $\mathrm{PhSeBr}$ ) in the PS- $b$-P4VP system, the amphiphilicity of the polymer is altered and the emulsion could change from an $\mathrm{O} / \mathrm{W}$ single emulsion to a W/O/W double emulsion. After solvent evaporation, the $\mathrm{W} / \mathrm{O} / \mathrm{W}$ double emulsion could provide porous solid microparticles, which can function as selective artificial GPx mimics. This 
methodology is promising to find applications in drug delivery, catalysis and many other areas.
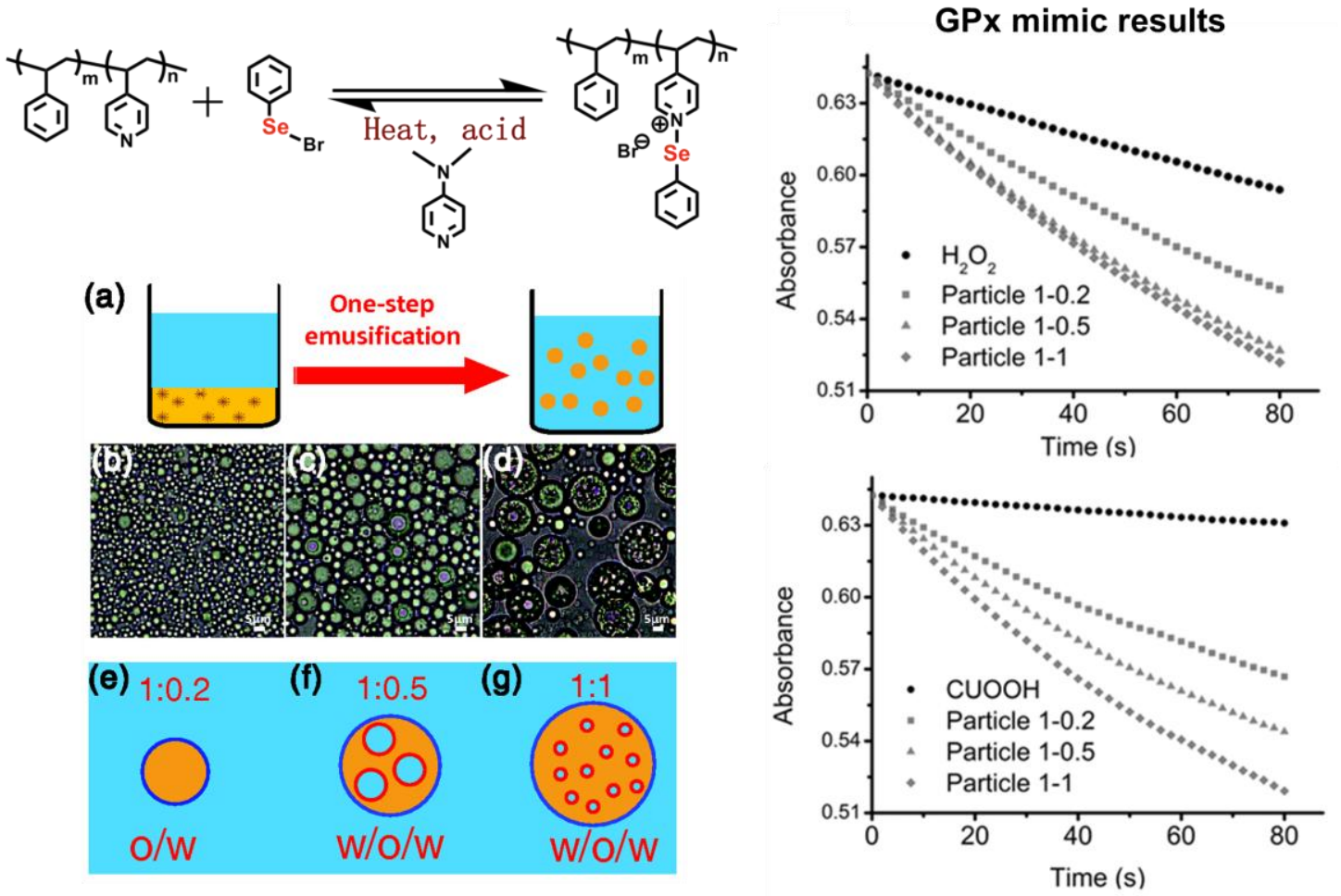

Fig. 10 Tuning polymeric amphiphilicity via Se-N interactions: towards one-step double emulsion for highly selective enzyme mimics. With $\mathrm{PhSeBr}$ gradually added in the PS- $b$-P4VP system, the emulsion could change from $\mathrm{O} / \mathrm{W}$ single emulsion to $\mathrm{W} / \mathrm{O} / \mathrm{W}$ double emulsion. The right figure indicated the solid microparticles after solution evaporation could be used as GPx mimics [55].

\section{$\gamma$-ray responsive materials}

$\gamma$-ray is one of the most widely used ionizing radiations for clinical radiotherapy because of its deep penetrating ability and strong power [56]. Radiotherapy is to use ionizing radiation to control or kill the malignant cells, while chemotherapy is the 
treatment of cancer with chemotherapeutic agents [57-59]. As conventional chemotherapy and radiotherapy both suffer from low efficiency and side effects, we propose the idea of combining chemo- and radio- therapies to afford better efficiency and fewer side effects [60].

Radiation chemistry revealed that ROS could be generated if water was irradiated by ionizing radiation [61]. We anticipated that the ROS produced by $\gamma$-ray radiation may be able to trigger the response of smart delivery vehicles. If anticancer therapeutics were loaded into the self-assembled vehicles and released at the site of irradiation, combined chemo- and radio-therapies could be achieved. Since ionizing radiation is also hazardous, the responsive systems need to be ultra-sensitive to oxidation so as to reduce the dosage of $\gamma$-ray employed.

Taking advantage of the sensitive nature of diselenide bonds, our group fabricated a $\gamma$-ray responsive hydrogel by the complexation of a positively charged diselenide-containing polymer and a peptide amphiphile (Fig. 11) [62]. The two building blocks could complex together and form long crosslinked nano-fiber networks. When irradiated by $0.5 \mathrm{kGy} \gamma$-ray, the long fibers tended to get shorter and eventually the network and the hydrogel structure was disrupted, resulting in a gel-sol transition. The radiation-generated ROS in water could cause the oxidative cleavage of the Se-Se bond on the polymer backbone, which accounts for the response $\gamma$-ray. The GPC trace of the irradiated sample confirmed the proposed mechanism since no 
polymer signal could be spotted. As a control, the $\gamma$-ray response of a similar disulfide-containing polymer hydrogel was quite different, as it remained intact after 5 kGy radiation. It means that the active diselenide bonds are crucial to the $\gamma$-ray responsiveness. However, its ability to undergo gel-sol transition only at relatively high $\gamma$-ray dosages presents challenges for practical biomedical or bioanalytical applications.
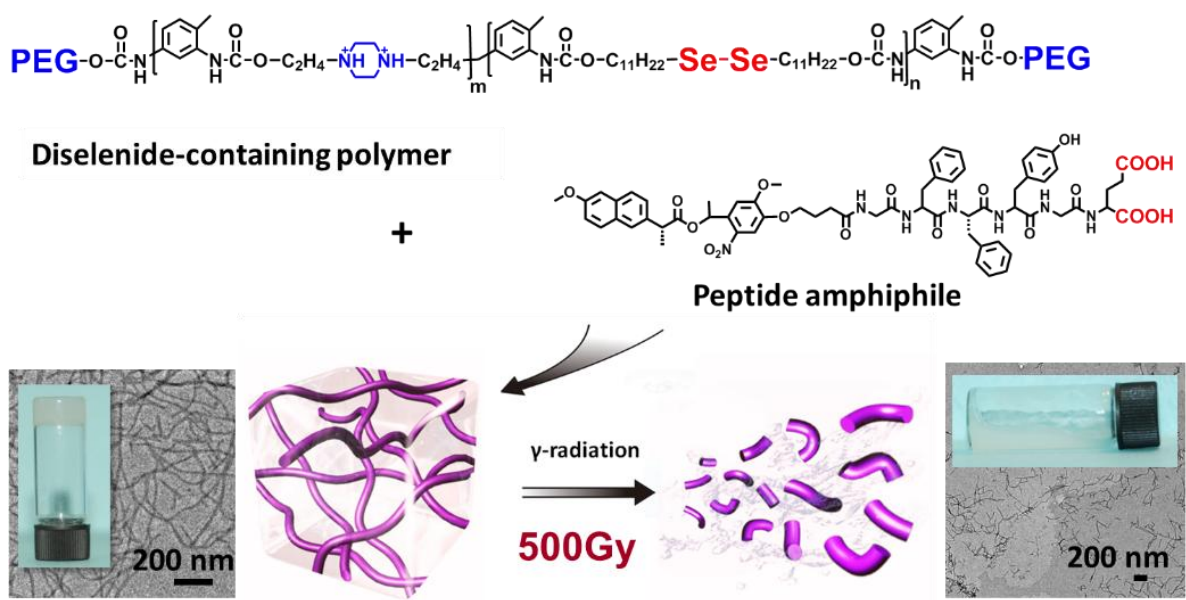

Peptide amphiphile

Fig. $11 \gamma$-ray responsive hydrogel formed by a diselenide-containing positively charged polymer and a peptide amphiphile. The hydrogel was formed by long nano-fibers, as shown in the left figure. In the presence of $500 \mathrm{~Gy} \gamma$ ray, the long nano fibers tended to get shorter and the hydrogel network was disrupted. Copyright 2013 WILEY-VCH [62].

The efficacy of $\gamma$-ray response is limited by the transportation kinetics of the ROS generated. In the hydrogel, the densely crosslinked nanofiber and immobilized water made it difficult for the ROS to diffuse and react with the diselenide bonds. It is anticipated that if the diselenide bonds could be exposed in a free aqueous solution, 
the reaction efficiency may see a significant increase. Diselenide-containing polymer micelles were prepared with Dox loaded inside and treated with $\gamma$-ray of different dosages (Fig. 12) [63, 64]. $\gamma$-ray induced morphology change could be observed with clear dose effects. The micelles swelled slightly with 5 Gy $\gamma$-ray radiation and began to collapse into irregular aggregates when the dosage was increased to $50 \mathrm{~Gy} .500 \mathrm{~Gy}$ radiation could break almost all the micelles into irregular aggregates, indicating that all the micelles has been destroyed. In addition, the release profiles also suggested that even with low dosages of radiation, decent amounts of Dox could still be released. For example, about $45 \%$ Dox could be released in the presence of 5 Gy $\gamma$-ray. The rational designed example shows how the $\gamma$-ray response could be modulated for self-assembled nanostructures.

a)

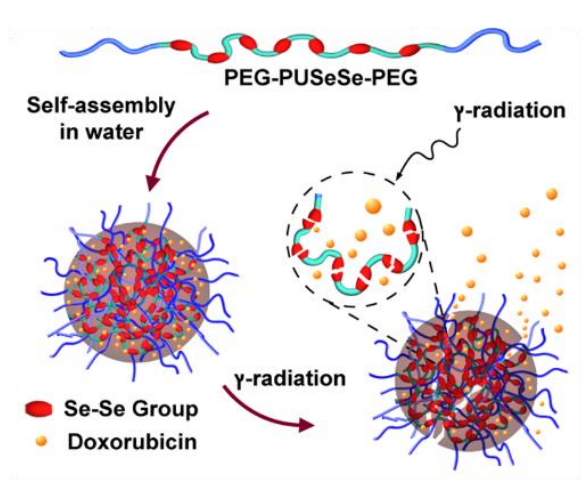

b)

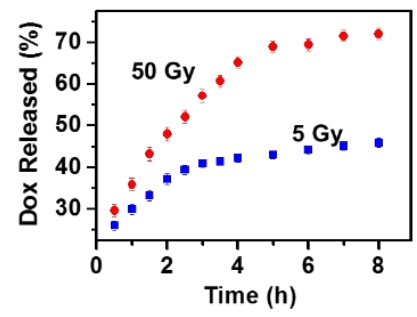

c)

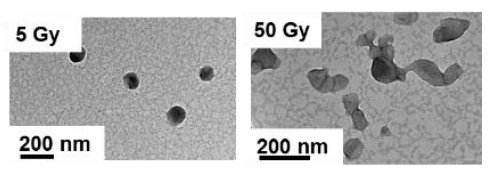

Fig. 12 The $\gamma$-ray responsive diselenide-containing polymer micelles. The micelles could afford controlled release of Dox with increased release percentage under increased $\gamma$-ray dosage (Fig b). The morphology of the micelles could also change after $\gamma$-ray radiation, as shown in the TEM images in Fig c. Copyright 2011 American Chemical Society [63]. 
Nevertheless, the systems above were still found wanting when considered for real clinical applications. New materials with enhanced sensitivity to $\gamma$-ray are highly demanding. In principle, materials with better ROS sensitivity would have better $\gamma$-ray response. Hence, the element tellurium should be a more promising candidate. The tellurium-containing polymer micelles were found to be highly responsive to $\gamma$-ray [43]. The micelles swelled from $40 \mathrm{~nm}$ to $250 \mathrm{~nm}$ within $1 \mathrm{~h}$ after $\gamma$-ray radiation for only 2 Gy by ${ }^{60} \mathrm{Co}$ radiation source. It is notable that the dosage a patient can receive one day for clinical radio-therpy is just 2 Gy [65]. The introdution of this tellurium-containing polymer will not only be a major advancement in ROS sensitive material, but also push forward the development of smart systems applicable for the combination of chemo- and radio- therapies.

\section{Coordination responsive materials}

Coordination responsive systems refer to systems responsive to the competitive coordination ligands and could subsequently achieve controlled release of loaded cargoes. Nature has demonstrated many examples of competitive interactions in metabolism processes. They play important roles in physiological activities, for example, the function of $\mathrm{G}$ protein-coupled receptors, the selective binding of antibodies with the immune globulin [66-68]. Utilizing biological ligands to modulate the properties of synthetic systems presents an interesting direction for the design of controlled delivery vehicles. Several advantages are obvious for coordination 
responsive systems. Numerous ligands are present in the physiological environment and the system also holds promises to work as multi-drug delivery system for synergetic therapies.

A coordination-responsive system was made by complexing platinum cations with a monoselenide-containing polymer (Fig. 13) [69]. We studied the coordination of selenium and platinum using model compounds and found that $\mathrm{Se}$ and $\mathrm{Pt}$ coordinate together with 3:1 stoichiometry. Doxorubicin could be loaded in the coordination micelles and released controllably through the competitive coordination by GSH. The platinum could be extracted from the polymeric micelles and the disassembly of platinum from the micelles could further trigger the release of Dox. To further verify the competitive-coordination-responsive mechanism proposed, DTT was employed as a stronger competitive ligand and both the Dox release rate and the release percentage were enhanced. Since platinum based drugs exist widely in clinical use, this coordination responsive system could serve as a multi-drug delivery system for cooperative chemotherapy. 

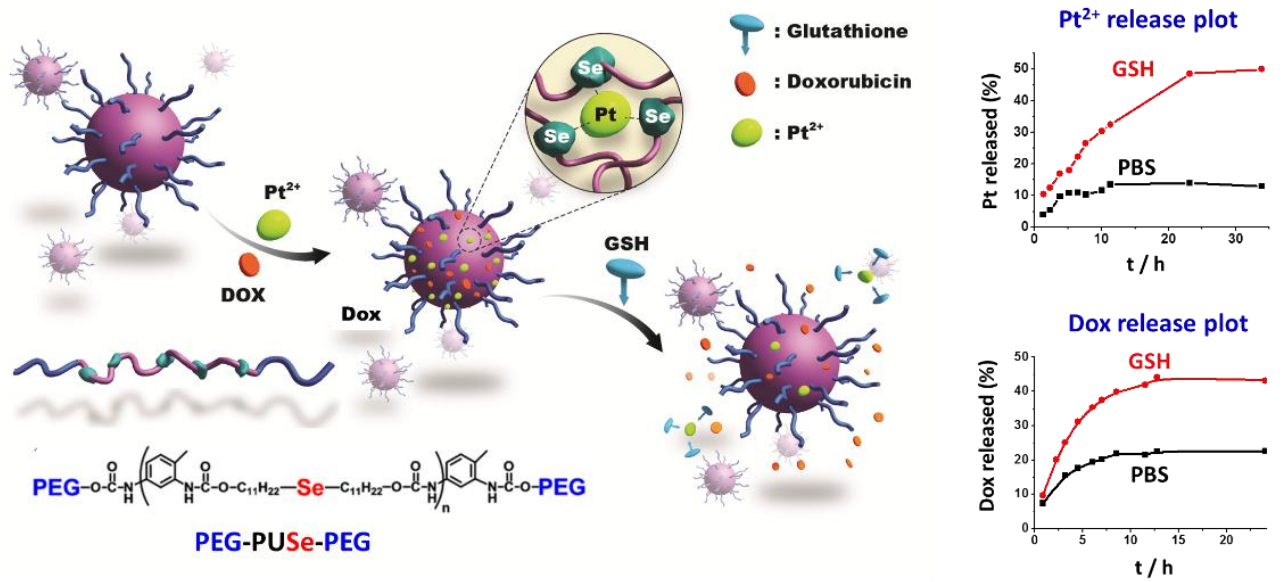

Fig. 13 Coordination-responsive delivery system formed by selenium-containing polymer and platinum cations. Dox could be loaded in the coordination micelles. In the presence of the competitive ligand GSH, platinum cations could disassemble from the micelles and subsequently trigger the release of Dox. Copyright 2012 Royal Society of Chemistry [69].

To further see if the coordination responsive systems could be used for clinical platinum drugs, we seek to improve the system by introducing better coordinating moieties into polymers. According to the Pearson acid base concept, tellurium is a softer base compared to selenium and therefore should coordinate better with cisplatin. Tellurium was introduced into water soluble polymers as drug delivery vehicles for the first time (Fig. 14) [70]. The coordination chemistry between tellurium and platinum was designed to enable the polymers to load platinum drugs. Through the competitive coordination of biomolecules, the drugs could be released in a controlled manner. Furthermore, the release process showed a ligand-regulated manner. The release kinetics could be modulated by different competitive ligands such as GSH, 
DTT and spermine. The advantage of the new coordination triggered release is that release kinetics could be modulated for time spans ranging from several days to as long as a month. As a result, the goal of enhanced delivery efficiency and fewer side effects may be achieved. Spermine is one of the polyamine with strongest biological activities and exists widely in the human body. Being closely related to cell proliferation, elevated spermine concentration is also regarded as biomarker for rapidly growing tumor. This coordination responsive system provides a new possibility to combine the glutathione response, which is mostly based on its reductivity, with the spermine response, therefore making it more convenient for synergetic multi-responsive smart systems.

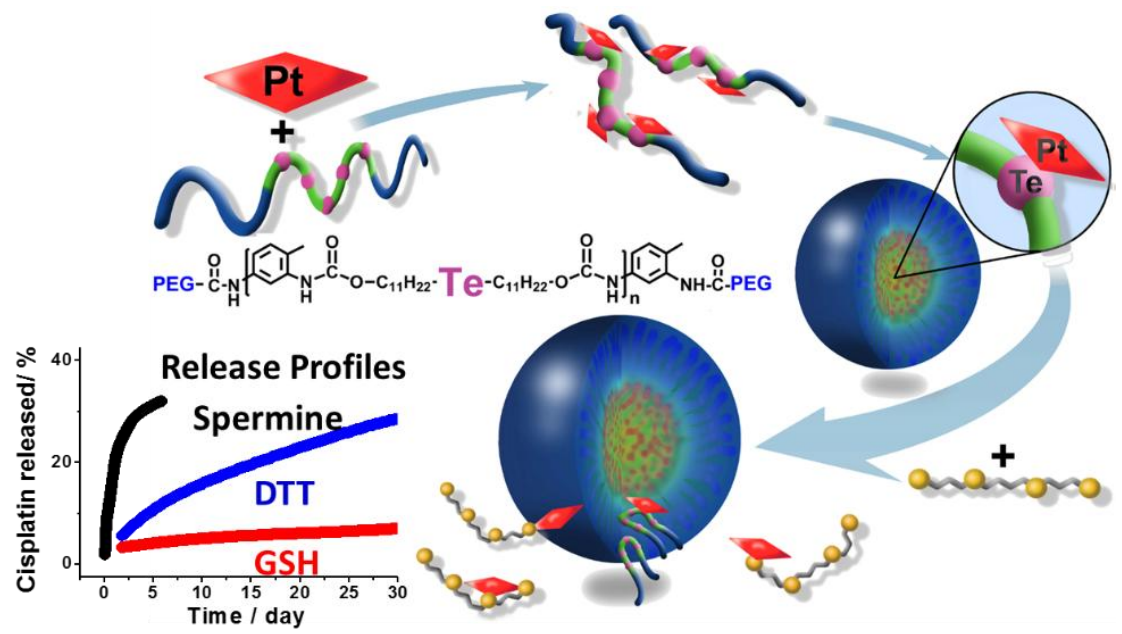

Fig. 14 Coordination-responsive drug delivery system based on tellurium-containing polymer. Telluride monomer was utilized to enable better coordination with cisplatin. Different ligands could lead to different release profiles over different time spans. Copyright 2014 American Chemical Society [70]. 

approaches that could tune the release of payload. It is assumed that the cisplatin first dissociate from the polymer backbone, and then diffuse out of the hydrophobic core followed by the hydrophilic PEG shell, before finally being released from the delivery vehicle. We anticipate that if the diffusion process could be shortened, the release rate could be enhanced. Based on this design, a LbL film was fabricated by alternatively depositing diazo-resin (DAR) and a cisplatin-coordinated tellurium-containing compound (Fig. 15) [71]. Stable films with $80 \mathrm{~nm}$ in thickness were fabricated by photo crosslinking after LbL growth. As a result of its thinness, the release of cisplatin from the films was much faster when treated with competitive molecules such as spermine or spermidine. Considerable amount of cisplatin could be released within 1 h. This example suggests that the competitive coordination system is robust and could be fabricated not only in solution, but also on surface. It shows how structure engineering could be used to tune the performance of coordination-responsive controlled delivery systems as well. 
a)

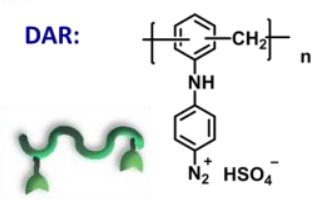

M-TeCOOH:

$\mathrm{HOOC}-\mathrm{C}_{7} \mathrm{H}_{14}-\mathrm{Te}-\mathrm{C}_{7} \mathrm{H}_{14}-\mathrm{COOH}$

LbL assembly and UV radiation

cisplatin:
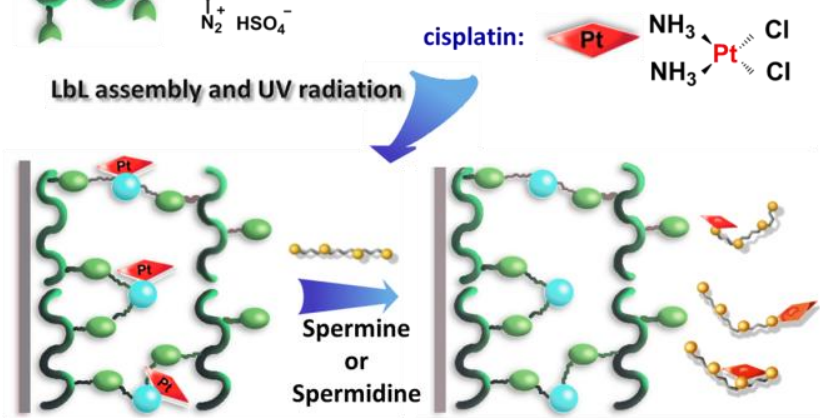

b)

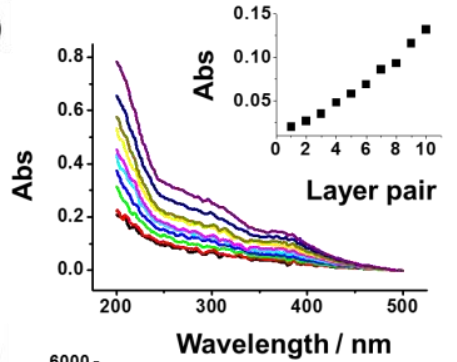

c)

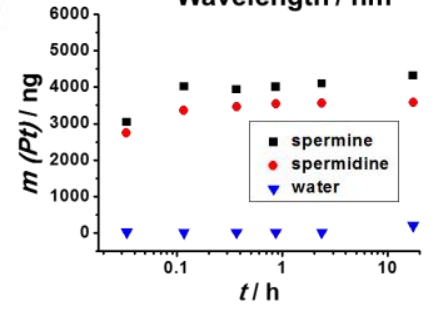

Fig. 15 Telluride-compound/cisplatin containing LbL multilayer films on the interface with coordination-responsive release behaviors. The LbL assembly was performed in a controlled manner (Fig b). The release kinetics could be further modulated by the different competitive ligands such as spermine and spermidine (Fig c). Copyright 2015 Royal Society of Chemistry [71].

\section{Materials that can tune ROS level}

Reactive oxygen species (ROS), such as hydrogen peroxide $\left(\mathrm{H}_{2} \mathrm{O}_{2}\right)$, hypochlorite $\left(\mathrm{OCl}^{-}\right)$, superoxide $\left(\mathrm{O}^{-}{ }^{-}\right)$, and hydroxyl radical $\left({ }^{\bullet} \mathrm{OH}\right)$, together with reactive nitrogen species (RNS), such as nitric oxide $\left({ }^{\circ} \mathrm{NO}\right)$ and peroxynitrite $\left(\mathrm{ONOO}^{-}\right)$play a very crucial role in health [72]. Excess production of ROS or RNS, so-called oxidative stress, however, may also lead to diseases, such as cancer, atherosclerosis, cardiovascular disease and so on [73, 74]. Apart from their interesting chemical properties, selenium-containing compounds are also known for their close relation with ROS [75-77]. Seleno-enzyme GPx has been reported to be capable of catalyzing the release of $\mathrm{NO}$ from the decomposition of RSNOs both in vitro and in vivo [78]. 
NO (Nitric oxide) is an important cellular signalling molecule involved in many physiological and pathological processes in mammals. It is a powerful vasodilator which can facilitate vasculature relaxation and the inhibition of platelet adhesion and activation. S-nitrosothiols (RSNOs) serve to transmit NO bioactivity, acting as potential NO storage, transfer, and delivery vehicles that exist in blood and within living cells. Therefore it is important to develop reliable methods to detect RSNOs that can help diagnose vascular diseases as well as assess the benefits of potential treatments $[78,79]$.

Meyerhoff et al. have successfully prepared a RSNOs biosensor by utilizing an organoselenium catalytic polymer immobilized within a hydrogel layer at the surface of an amperemeter. The biosensor can be used to detect the presence and relative levels of RSNO species at $0.1 \mu \mathrm{M}$ levels within fresh blood samples, with sensor lifetimes of at least 10 days [80]. Besides, they have also reported the first NO generating material that utilizes immobilized organoselenium polymeric moieties as a catalyst for RSNO decomposition (Fig. 16) [81]. The diselenide-crosslinked polyethylenimine (PEI) and cellulose filter paper exhibit catalytic activity t RSNOs decomposition and can be used for NO generation from RSNOs. The diselenide-modified PEI hydrogel or cellulose membrane surfaces were shown to exhibit true catalytic activity in that NO generation is limited unless a thiol reducing agent is also present. The materials are expected to exhibit greatly enhanced 

blood-contacting devices.

(1) $\mathrm{RSe}-\mathrm{SeR}+\mathrm{GSH} \rightleftharpoons \mathrm{RSe}-\mathrm{SG}+\mathrm{RSe}-+\mathrm{H}^{+}$

(2) $\mathrm{RSe}-\mathrm{SG}+\mathrm{GSH} \rightleftharpoons \mathrm{RSe}-+\mathrm{H}^{+}+\mathrm{GS}-\mathrm{SG}$

a)
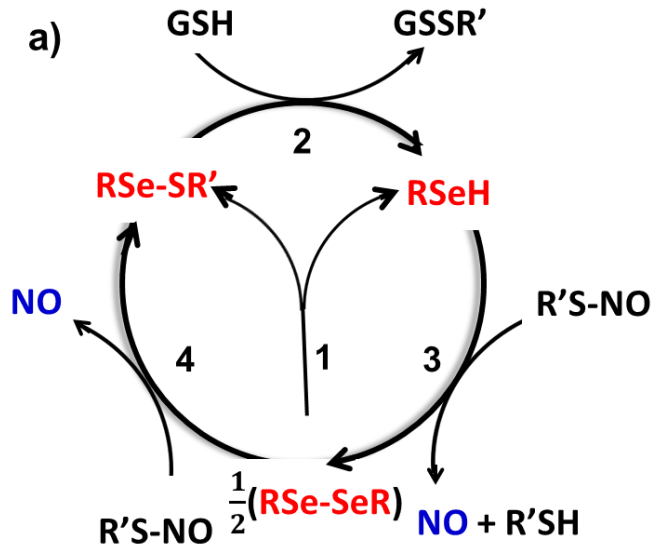

(3) $\mathrm{RSe}-+\mathrm{H}^{+}+\mathrm{R}^{\prime} \mathrm{S}-\mathrm{NO}=1 / 2 \mathrm{RSe}-\mathrm{SeR}+\mathrm{R}^{\prime} \mathrm{SH}+\mathrm{NO}$

(4) $\mathrm{RSe}-\mathrm{SeR}+2 \mathrm{R}$ 'S-NO $\rightleftharpoons 2 \mathrm{RSe}-\mathrm{SR}^{\prime}+2 \mathrm{NO}$ b)

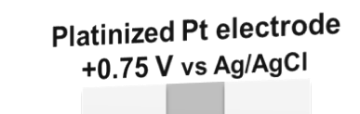

Internal

solution

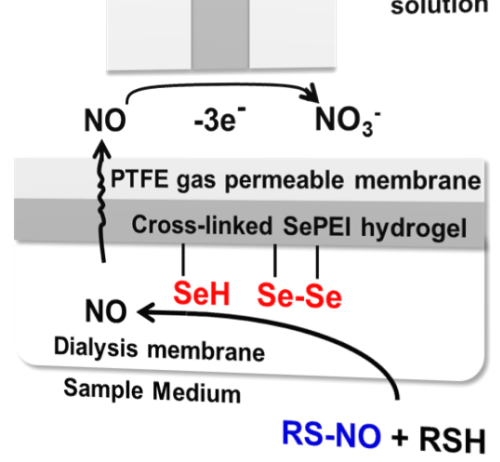

c) $\quad \mathrm{CH}_{2} \mathrm{CH}_{2} \mathrm{NH}_{2}$

$\left(\mathrm{CH}_{2} \mathrm{CH}_{2} \mathrm{~N}\right){ }_{y}\left(\mathrm{CH}_{2} \mathrm{CH}_{2} \mathrm{NH}\right)\left(\mathrm{CH}_{2} \mathrm{CH}_{2} \mathrm{~N}\right)$
$x-\mathrm{Se} \mathrm{Se}-\mathrm{R} y^{\prime}$
$\left(\mathrm{H}_{2} \mathrm{CH}_{2} \mathrm{C}-\mathrm{N}\right)_{y^{\prime}}\left(\mathrm{CH}_{2} \mathrm{CH}_{2} \mathrm{~N}\right)_{y}\left(\mathrm{CH}_{2} \mathrm{CH}_{2} \mathrm{NH}\right)_{x}$
SePEI $\quad \mathrm{CH}_{2} \mathrm{CH}_{2} \mathrm{NH}_{2}$

Fig.16 Organoselenium polymeric moieties for the generation of NO and the detection of S-Nitrosothiol. a) Scheme of the proposed RSNO decomposition reactions by organoselenium catalyst using glutathione as reducing agent b) Amperometric detection scheme of proposed RSNO sensor based on catalytic RSe-hydrogel layer; (c) chemical structure of SePEI [81].

Later on the same group have developed a method for immobilizing catalytic organoselenium species to blood-contacting biomedical devices by LbL assembly of positively charged diselenide crosslinked PEI or Ebselen-attached PEI and negatively 
charged sodium alginate [82]. The capability of the multilayer films to generate NO fluxes can be finely tuned by the number of layers. The film stability can be enhanced via self-annealing and cross-linking, exhibiting minimal selenium leaching to the solution. The Ebselen-based LbL films are able to produce physiological levels of NO from RSNOs after soaking in blood even for $24 \mathrm{~h}$. In addition, these LbL fims are able to prevent surface attachment by living bacterial and show promising broad-spectrum antimicrobial effects. Since the selenium-containing polymer films exhibit both antithrombotic and antimicrobial properties, these materials may find applications to resolve complications due to thrombus formation and bacterial infection associated with a wide range of biomedical devices $[83,84]$.

Our group reported a new drug based on the coordination of a selenium-containing small molecule EgSe and cisplatin, which possesses ROS-mediated selective anti-cancer effects (Fig. 17) [85]. The selenium-containing drug could kill cancer cells in vitro with significant selectivity. The viability of L-02 cells remained $72 \%$ whereas only $4 \%$ of the cancer cells (HepG2 cells) was alive. Detailed analysis revealed the intracellular concentration of Pt in HepG2 cells was much higher than L-02 cells and that the amount of ROS was also much higher. The clearly similar trend in the ROS concentration and the rate of apoptosis indicated that the highly selective anticancer effects was caused by the ROS-induced apoptosis, which functions differently from the parent drug cisplatin. The drug was also found to have broad-spectrum anticancer activities. In vivo experiments on tumor-bearing mice 
showed that the drug could effectively control the growth of the tumor without causing any weight loss. Another selenium containing compound with three methyl groups was designed and synthesized [86]. EgSe-tMe had weaker coordination with cisplatin and therefore had no selectivity towards cancer cells and normal cells. However, such selectivity could be remained when cisplatin was replaced by $\mathrm{PtCl}_{2}$, which indicated that cisplatin was not necessary for selective anticancer activity and that the coordination bond is crucial to the system. This method revealed that the coordination compound between selenium-containing molecule and cisplatin could be used to selectively kill cancer cells through an ROS-induced mechanism.

a)

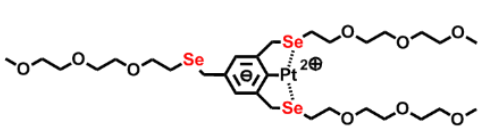

b)

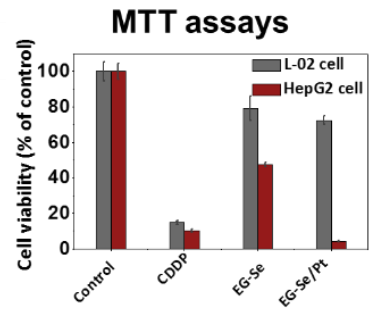

c)

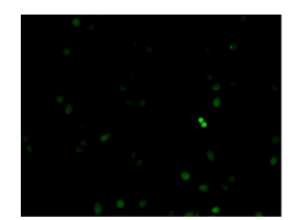

d)

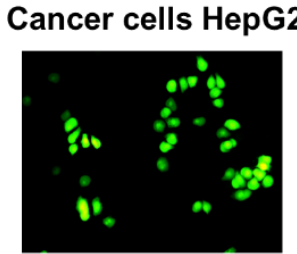

e)

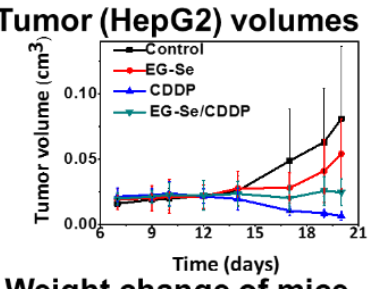

f)
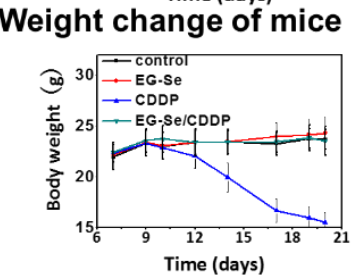

Fig. 17 Selenium-platinum coordination compound as novel anticancer drug. The drug was prepared by the coordination of EgSe and cisplatin, as shown in Fig a. It could selectively kill the cancer cells (Fig b) with different viability from cisplatin. As shown by the ROS fluorescence probe experiments, the ROS level in cancer cells after treated with the drug are much higher than normal ones (Fig c, d). The drug could effectively control the growth of tumor in vivo (Fig e) without decreasing the 
weight of the mice (Fig f) [85].

\begin{abstract}
A variety of complex selenium-containing nano-materials can also find applications in mediating ROS level. Chen et al. reported a mesoporous silica nanoparticle (MSN) as carriers for the anticancer selenocystine to achieve syngetic erchemo-/radiotherapy [87]. Selenocystine (Sec) is a novel selenocompound with anticancer potency and could be released from the MSNs in the presence of lysozyme at low pH. SeC@MSNs significantly enhanced X-ray induced growth inhibition in cervical cancer cells. Upon radiation by X-ray, SeC@MSNs promoted intracellular ROS overproduction and subsequently caused DNA damage and cell death, thus achieving enhanced anticancer efficacy. This is an example showing that selenium compound could be used to enhance X-ray induced ROS overproduction, further causing cancer cell apoptosis. They also fabricated targeting ligand conjugated selenium inorganic nanoparticles (SeNPs) as drug delivery vehicles (Fig. 18) [88]. The Dox-loaded SeNPs showed enhanced cytotoxicity toward cancer cells in vitro by activating p53 and MAPKs pathways to promote cell apoptosis. While in vivo experiments in nude mice model also indicated that the system could inhibit the growth of tumor.
\end{abstract}


a)

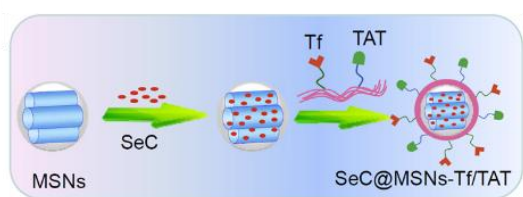

b)

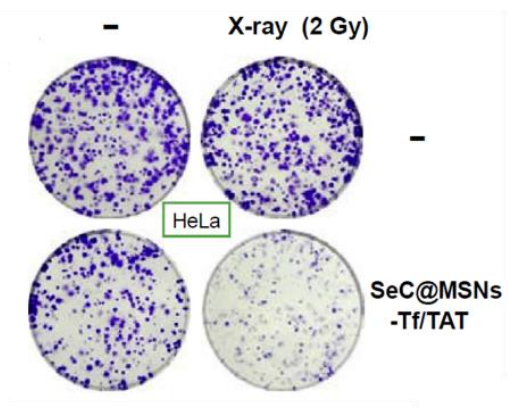

c)
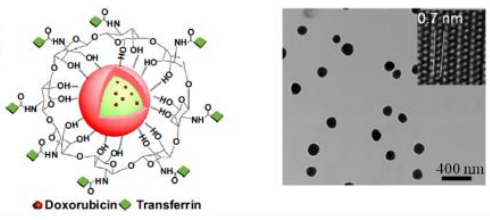

d)

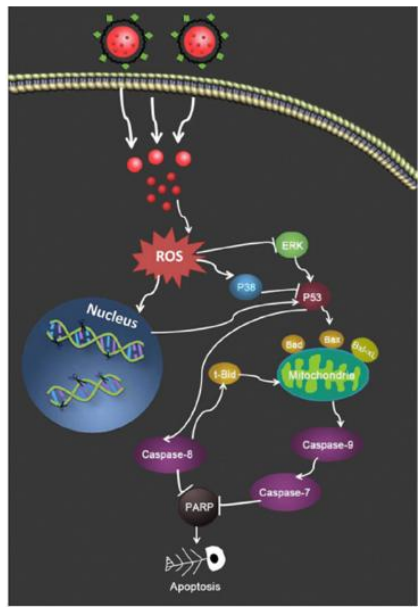

Fig. 18 Composite selenium-containing nanoparticles for tuning the intracellular ROS level. The left is the SeC@MSNs-Tf/TAT for the synergetic cancer chemo-/radiotherapy. The structure (Fig a) and the image of HeLa cell colony formation (Fig b) indicated the enhanced inhibitory effects of X-ray on the colony formation. The right is the cancer-targeted selenium nanoparticles (Fig c). The internalization of the selenium nanoparticles triggered ROS production and activate p53 and MAPKs pathways to the promote apoptosis [88].

\section{Gene delivery vector and self-delivery therapeutic materials}

Gene delivery, the process of introducing foreign DNA into host cells, is one of the necessary steps for gene therapy $[89,90]$. Recently, the complexation of cationic polymers and gene to form polymeric gene carriers has emerged as a promising non-viral delivery strategy, owing to its ease of manufacturing, ability to carry DNAs 
of indefinite size, less inflammatory response, and lack of immunogenicity. One important approach is to design biodegradable cationic polymers which allow efficient gene transfer and stimuli-responsive degradation into low molecular products with minimal toxicity.

Recently, the diselenide-bonds has been introduced into gene carrier systems. $\mathrm{Gu}$ et al. successfully designed and synthesized cationic polymers as gene carriers with diselenide-bonds cross-linked to branched oligoethylenimine (OEI-SeSe) [91]. This cross-linked $\mathrm{OEI}_{800}-\mathrm{SeSe}_{\mathrm{x}}$ has high molecular weight and can efficiently compact with plasmid DNA (pDNA) to form cationic complexes. After entering an environment of high GSH concentration, such as the cytoplasm, the diselenide bonds of $\mathrm{OEI}_{800}-\mathrm{SeSe}_{\mathrm{x}}$ can be cleaved, breaking into subunits of $\mathrm{OEI}_{800}$ and leading to disassembly of the cationic complexes. This process helped to transfer plasmid DNA into the nucleus for transcription. $\mathrm{OEI}_{800}-\mathrm{SeSe}_{\mathrm{x}}$ showed both much lower cytotoxicity than $\mathrm{PEI}_{25 \mathrm{k}}$ and significantly higher transfection efficiency than $\mathrm{OEI}_{800}$ in $\mathrm{B} 16 \mathrm{~F} 10$ and HeLa cells. Thus diselenide-containing polycationic systems have been proven to be suitable for reduction-sensitive gene delivery.

After that, $\mathrm{Cai}$ and $\mathrm{Gu}$ et al. studied the differences between diselenide-containing $\left(\mathrm{OEI}_{800}-\mathrm{SeSe}_{\mathrm{x}}\right)$ and disulfide-containing $\left(\mathrm{OEI}_{800}-\mathrm{SS}_{\mathrm{x}}\right)$ cross-linked polymers with similar structure (Fig. 19) [92]. They revealed that both $\mathrm{OEI}_{800}-\mathrm{SeSe}_{\mathrm{x}}$ and $\mathrm{OEI}_{800}-\mathrm{SS}_{\mathrm{x}}$ with a high molecular weight could efficiently compact 
with pDNA into complexes. And diselenide bonds could be cleaved in the presence of $10 \mathrm{mM}$ GSH (tumor-relevant glutathione concentration), which is similar to the performance of disulfide bonds. But diselenide bonds in the cross-linked polymer are much more stable than disulfide bonds at $10 \mu \mathrm{M} \mathrm{GSH}$, a lower redox potential mimicking the extracellular GSH levels. These results indicated that the diselenide-containing gene vectors could be stable under extracellular conditions and undergo a rapid degradation in the intracellular reductive environment.
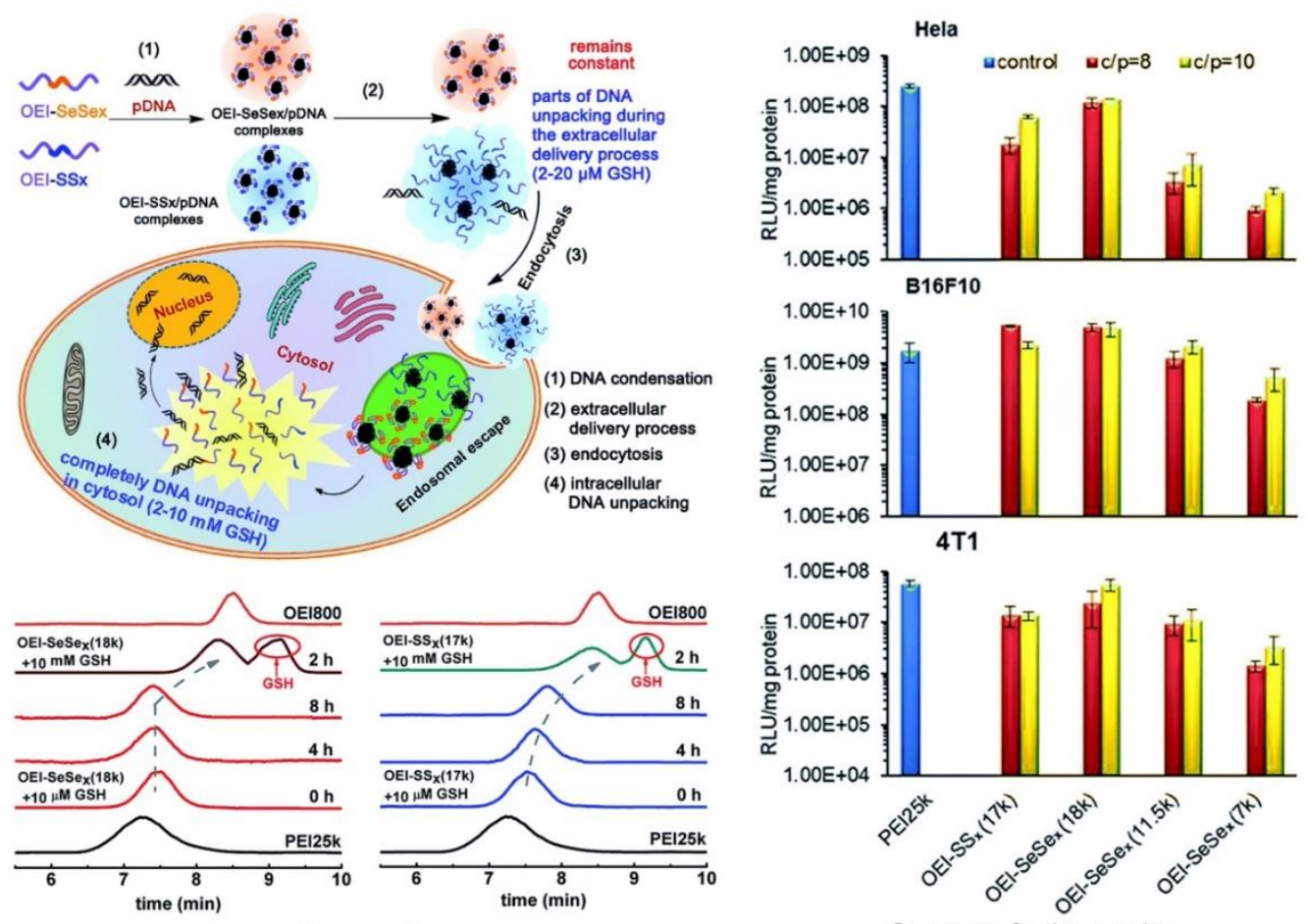

GPC results

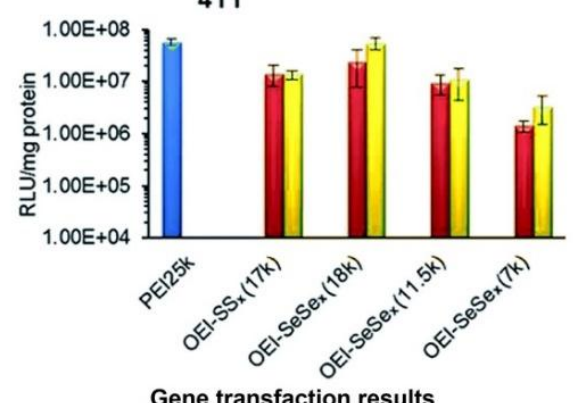

Fig. 19 The influence of reduction-sensitive diselenide bonds and disulfide bonds on oligoethylenimine conjugates for gene carrier. The load and the reduction trigged intracellular release of DNA. The lower left of the figure is GPC results of

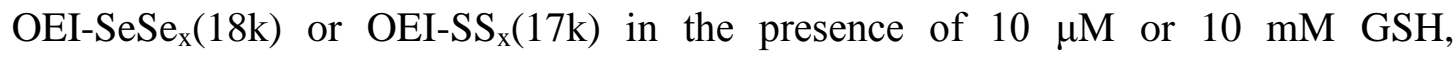
respectively. The right of the figure is the gene transfection results of Hela, B16F10 
and 4T1 cells, respectively. (Reproduced with permission of the Royal Society of Chemistry) [92].

Exploiting the difference between diselenide bonds and disulfide bonds in their responsiveness to reducing agents, $\mathrm{Gu}$ et al. designed a viral mimicking ternary polyplex as a vector for gene delivery, which could realize a reduction-controlled hierarchical unpacking process (Fig. 20) [93]. Diselenide-conjugated oligoethylenimine $\mathrm{OEI}_{800}-\mathrm{SeSe}_{\mathrm{x}}$ was firtstly utilized to compact DNA to form a nanoscale core, which was further encapsulated in disulfide-modified hyaluronic acid derivatives (denoted as HA-SS-COOH) to obtain ternary polyplexes (DNA/OEI-SeSe $\left.{ }_{x} / \mathrm{HA}-\mathrm{SS}-\mathrm{COOH}, \mathrm{DOS}\right)$. This system could achieve a stepwise responsiveness to the reduction gradients from the tumor extracellular environments to intracellular conditions. The negatively charged shell of HA-SS-COOH could protect the core in physiological conditions and DOS polyplexes could be internalized by cancer cells via receptor-mediated endocytosis. Next, the continuous unpacking was attributed to the different redox sensitivities of the disulfide bonds and the diselenide bonds. Gene transfection in BALB/c nude mice and HepG2 cells showed higher efficacy compared to that of DP (DNA/PEI) and DPH (DNA/PEI/HA), which indicated potential application of the system in gene delivery. The reason why disulfide bond is more sensitive to reduction is still unclear and remains to be clarified by more strictly controlled experiments or simulations. 


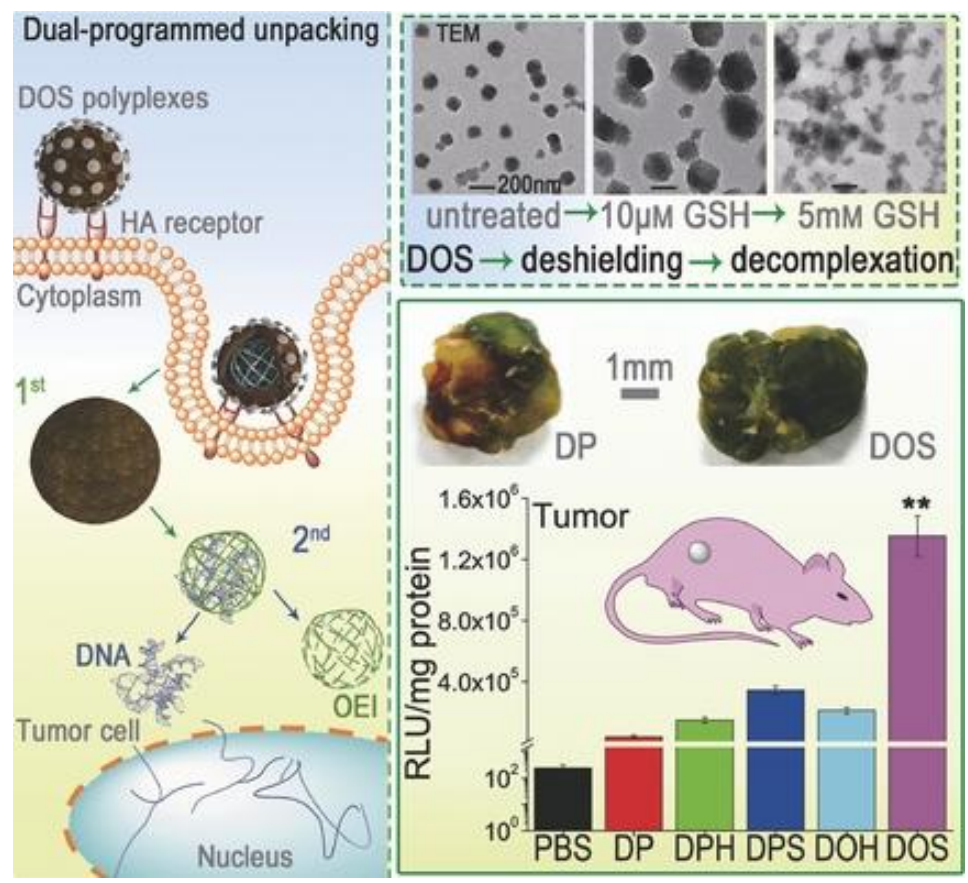

Fig. 20 Viral mimicking ternary polyplexes: a reduction-controlled hierarchical unpacking vector for gene delivery. Using OEI800-SeSe $\mathrm{x}_{\mathrm{x}}$ to compact DNA to form a nanoscale core, then further HA-SS-COOH to obtain ternary polyplexes (DNA/OEI-SeSex/HA-SS-COOH, DOS). The polyplexes could realize a stepwise reduction responsiveness to release DNA, as shown in the left of the figure. The upper right of the figure is the morphology of the polyplexes before and after treatment of different concentration of GSH. The lower right shows the gene transfection results [93].

Contrary to Gu's find, Wang et al. reported that the diselenide bonds were easier to be cleaved than disulfide bonds at GSH concentration of $0.3 \mathrm{mM}$ (Fig. 21) [94]. PEGylated polycations (mPEG-SeSe-PEI, mPEG-SS-PEI) were designed and synthesized as gene vectors. They studied the diameter of the particles in the presence of $0.3 \mathrm{mM} \mathrm{GSH}$ and the results showed the size of diselenide-containing PEGylated 
polyplexes underwent a more dramatic increase compared with disulfide-containing ones. Gene expression in HEK293T and HepG2 cells was higher with mPEG-SeSe-PEI/DNA polyplexes than with mPEG-SS-PEI/DNA, which can be attributed to more efficient endosomal escape ability by mPEG-SeSe-PEI/DNA polyplexes. However, the two examples cited above seemed to contradict from each other on the sensitivity of diselenide bond compared with that of disulfide bonds. Further control experiments and simulations are still needed to resolve the contradiction.

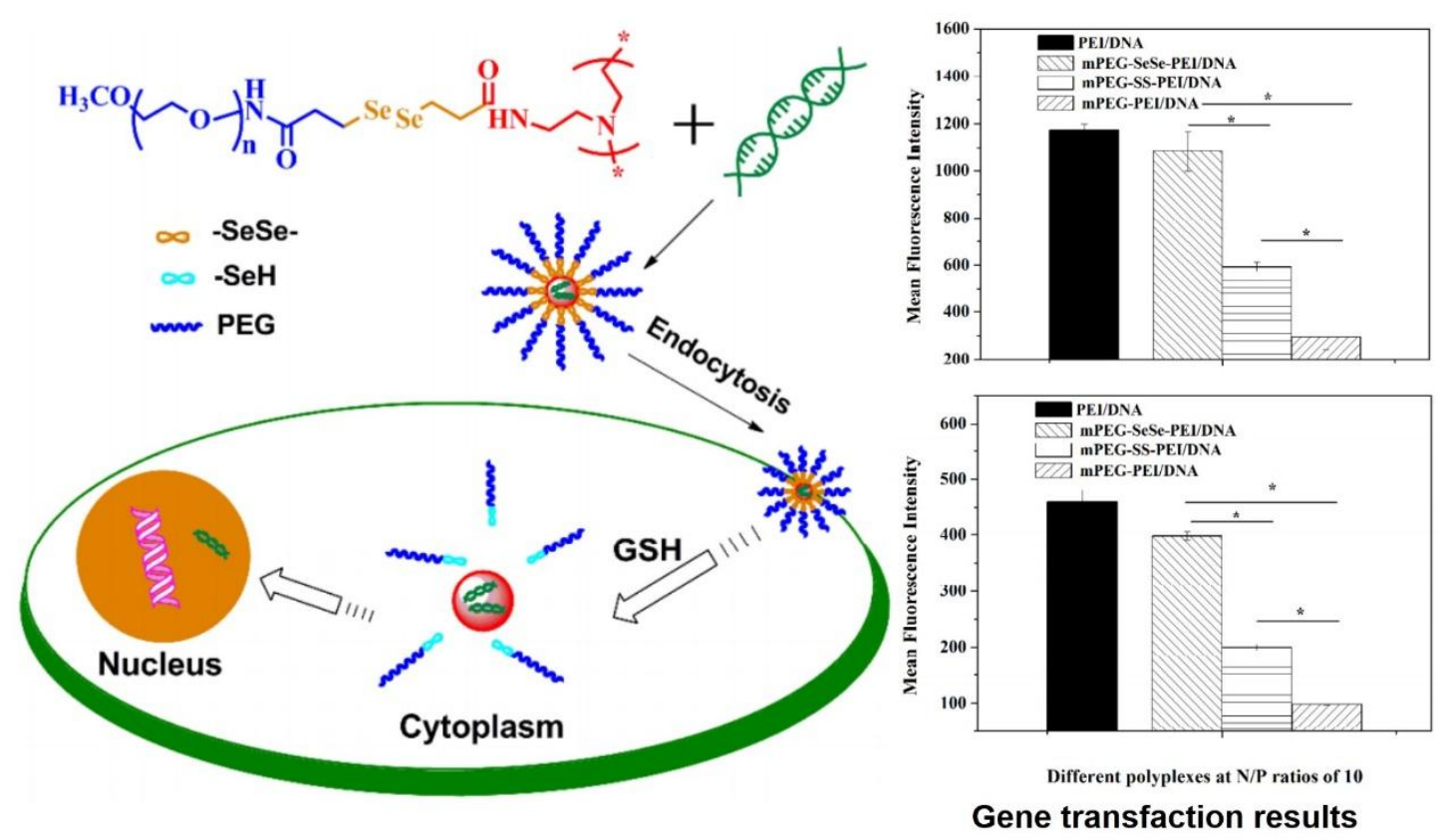

Fig. 21 Redox-triggered intracellular dePEGylation based on diselenide-linked polycations for DNA delivery. The right of the figure is pEGFP expression mediated by polycations/DNA polyplexes exposed to HEK293T cells (upper) and HepG2 cells (lower), respectively (Reproduced with permission of 2013 the Royal Society of Chemistry) [94]. 
When using polymer aggregates as delivery vehicles, the polymers themselves normally have no direct therapeutic effect and their metabolites might lead to unexpected side effects such as cytotoxicity and inflammation. It will be of significance to develop drug delivery vehicles that can not only encapsulate anticancer drugs in their aggregates but also exhibit anticancer properties by themselves, which will allow for the combination or cooperative chemotherapy. In the line of research into selenium-containing polymer nanomaterials, it is important to be aware that the selenium group itself may also have a functional role, in addition to being a structural moiety incorporated to facilitate self-assembly.

Huang, Yan et al. synthesized a hyperbranched polymer with alternative hydrophobic diselenide and hydrophilic phosphate groups (Fig. 22) [95]. The authors put more efforts on the study of anticancer property of Se itself and found that the hyperbranched polydiselenide exhibits good anticancer effect in a broad spectrum. Owing to the amphiphilic nature of the hyperbranched polydiselenide, it could self-assemble into micelles and then self-deliver into tumor cells through enhanced permeability and retention (EPR) effect. HPSeSe was obtained via an A2+B3 polymerization of diselenide-containing diol and phosphorous oxychloride, with an $\mathrm{M}_{\mathrm{w}}$ of $6100 \mathrm{~g} \mathrm{~mol}^{-1}$ (GPC). In vitro cytotoxicity and cell growth inhibition experiments indicated that HPSeSe specifically inhibited the proliferation of cancer cells, but induced no apparent toxicity effects on the viability of normal cells. 
Compared with conventional low-molecular-weight selenium anticancer agents, HPSeSe has significantly higher anticancer activity. And HPSeSe showed broad spectrum anticancer property, which the authors attributed to the synergistic effect of diselenide groups on the hyperbranced structures. Mechanism of the cancer cell death induced by HPSeSe could be traced to the caspase- 3 dependent apoptosis. In addition, HPSeSe could self-assemble to micelles and act as drug carrier to encapsulate Dox to realize responsive intracellular release upon GSH.
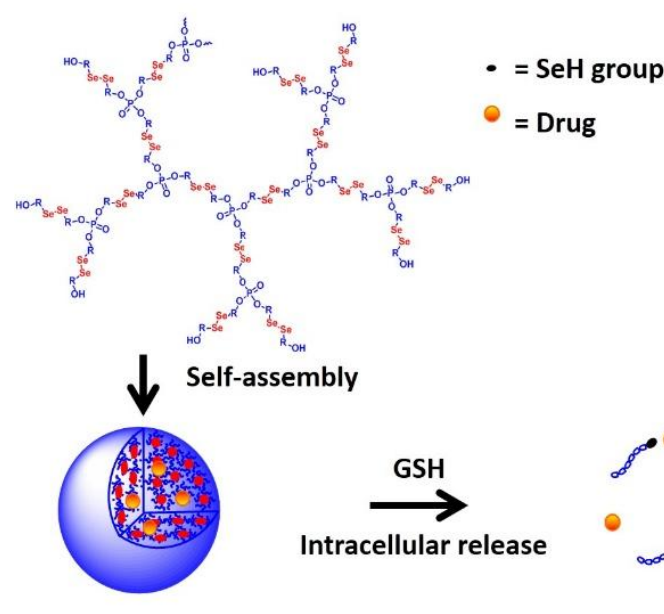
Elsevier) [95].

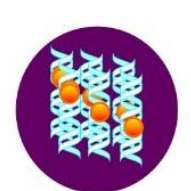

Nucleus uptake

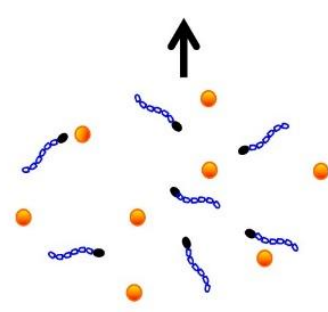

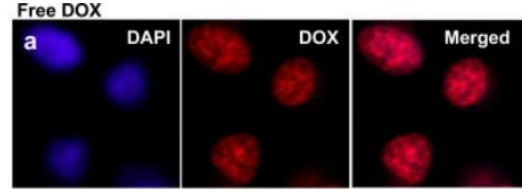

DOX-loaded HPSe micelles

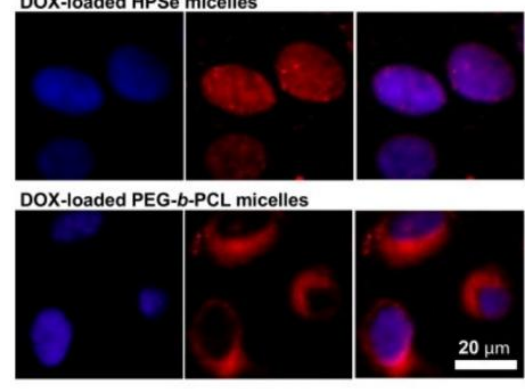

Drug delivery results

Fig. 22 Hyperbranched polydiselenide as a self-assembling broad spectrum anticancer agent: self-assembly of HPSeSe in water and its intracellular drug release triggered by GSH. The right of the figure is the drug delivery results of Hela cells incubated in the presence of free Dox, Dox-loaded HPSeSe micelles or Dox-loaded PEG-b-PCL micelles for $3 \mathrm{~h}$, respectively (Reproduced with permission of 2012

Another interesting example reported by Huang, Yan et al. is a hyperbranched 
polymer containing selenide and hydrophobic phosphate groups, which also exhibited good intrinsic anticancer effect. The anticancer property of selenium was further proved by using alkane- or oxygen-containing hyperbranched polymers as controls (Fig. 23) [96]. The authors synthesized a cyclic phosphate monomer by esterification of 2,2'-selenodiethanol with 2-chloro-2-oxo-1,3,2-dioxaphospholane. HPSe was synthesized via self-condensing ring-opening polymerization (SCROP) of the monomer, with a $\mathrm{M}_{\mathrm{n}}$ of $3800 \mathrm{~g} \mathrm{~mol}^{-1}$ (GPC). Due to the amphiphilic property of hyperbranched polyselenide (HPSe), it could self-assemble into spherical micelles with diameters of $70 \mathrm{~nm}$, which were appropriate for drug delivery via the EPR effect. Employing the ROS responsiveness of selenide, the hyperbranched polymer could be destructed rapidly within cancer cells and followed by the selective release of Dox. In vitro cell viability and flow cytometry experiments indicated that HPSe could specifically inhibit the proliferation of human cancer cells and the mechanism was mainly apoptosis.
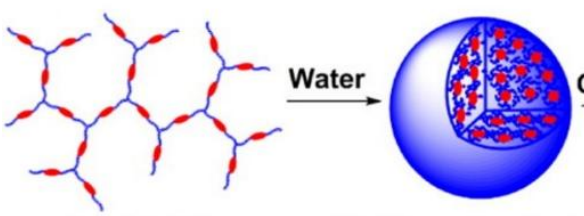

Oxidation Amphiphilic :-Se-

Multi-core/shell micelle
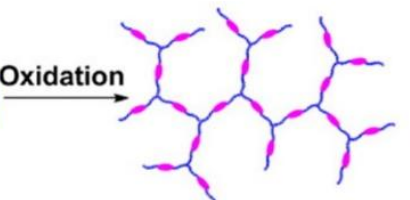
Hydrophilic - 0 $-\mathrm{Se}$
$-\ldots . .0$

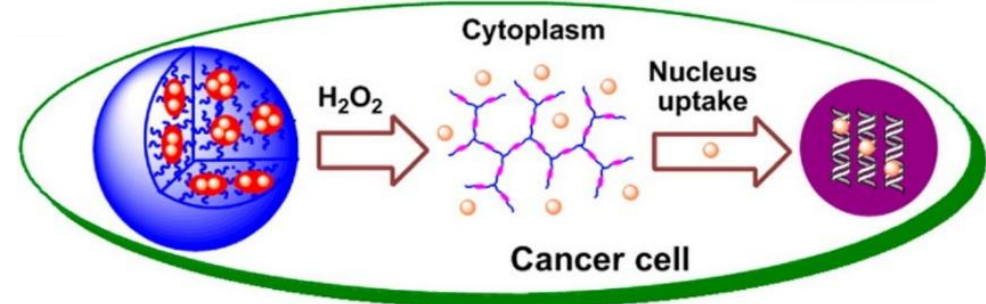

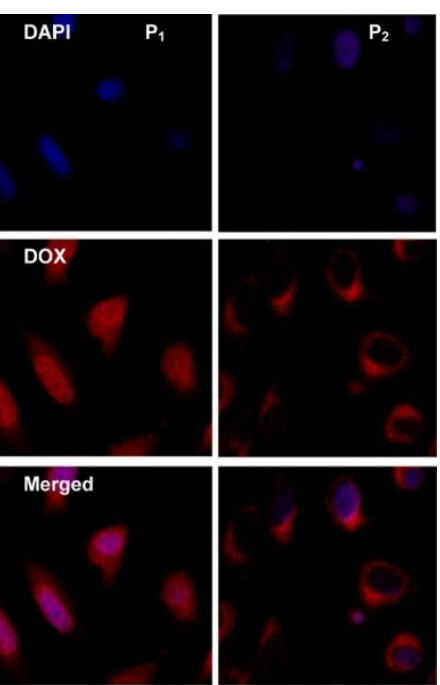

Drug delivery results 
Fig. 23 Therapeutic nanocarriers with hydrogen peroxide-triggered drug release for cancer treatment: self-assembly of HPSe in water and its intracellular drug release triggered by $\mathrm{H}_{2} \mathrm{O}_{2}$. The right of the figure is the drug delivery results of Hela cells incubated in the presence of Dox-loaded P1 micelles (Se-containing, left) and P2 micelles $\left(\mathrm{CH}_{2}\right.$-containing, right) for $3 \mathrm{~h}$, respectively (Reproduced with permission of 2013 the American Chemical Society) [96].

\section{Conclusions}

It is exciting to expect how much influence the selenium and tellurium polymer chemistry can have on the rational design and functional performance of the materials of future for nanotechnology and biomedical applications. Despite all the exciting recent progresses in nanomaterials, we cannot deny the fact that there is still a large gap between the intriguing systems reported in journals and commercially available materials for biomedical applications. As an emergent research area, the selenium/tellurium-containing nano-assemblies are not only of chemical interests by providing a series of unique stimuli-responsive properties, but also possessing with potential biological functions. The detailed biological activities of selenium/tellurium-containing assemblies are still in need of in-depth research. Parallel to the work on the at-equilibrium self-assembly, another important frontier is the fabrication of far-from equilibrium selenium/tellurium-containing assemblies that need sustained energy supply. Selenium/tellurium compounds are active electron donors and their reactions are potentially reversible, making them suitable to mimic 
the natural redox-involved metabolism.

\section{Acknowledgments}

This work was financially supported by the National Basic Research Program of China (2013CB834502), National Science Foundation for Distinguished Young Scholars (21425416), the National Natural Science Foundation of China (91427301), the Foundation for Innovative Research Groups of the National Natural Science Foundation of China (21421064). The authors thank Ke Qin for editing the language in this manuscript. Research on Se-polymers in Tsinghua was first initiated while Huaping Xu was working with Prof. Xi Zhang. The authors thank Prof. Zhang for his stimulating suggestions, discussions and continuous help during the past years.

\section{References}

[1] E.S.J. Arner, History of Selenium Research, 2012.

[2] C.K.N. Patel, Phys. Rev. Lett., 15 (1965) 1027-1030.

[3] J.T. Rotruck, A.L. Pope, H.E. Ganther, A.B. Swanson, D.G. Hafeman, W.G. Hoekstra, Science, 179 (1973) $588-590$.

[4] G. Mugesh, H.B. Singh, Chem. Soc. Rev., 29 (2000) 347-357.

[5] G. Mugesh, W.-W. du Mont, H. Sies, Chem. Rev., 101 (2001) 2125-2180.

[6] M.G. Barker, Coord. Chem. Rev., 103 (1990) 162-236.

[7] K. Huang, H. Xu, Selenium: Its Chemistry, Biochemistry and Application in Life Science (in Chinese), Huazhong Univ. Sci. Tech. Press2009. 
[8] C.W. Nogueira, G. Zeni, J.B.T. Rocha, Chem. Rev., 104 (2004) 6255-6286.

[9] L. Engman, Acc. Chem. Res., 18 (1985) 274-279.

[10] N. Petragnani, H.A. Stefani, Tetrahedron, 61 (2005) 1613-1679.

[11] X. Huang, X. Liu, Q. Luo, J. Liu, J. Shen, Chem. Soc. Rev., 40 (2011) 1171-1184.

[12] D.E. Discher, A. Eisenberg, Science, 297 (2002) 967-973.

[13] G. Riess, Prog. Polym. Sci., 28 (2003) 1107-1170.

[14] Y. Liu, B. Liu, Z. Nie, Nano Today, 10 (2015) 278-300.

[15] L. Zhang, F.X. Gu, J.M. Chan, A.Z. Wang, R.S. Langer, O.C. Farokhzad, Clinical Pharmacology \& Therapeutics, 83 (2008) 761-769.

[16] H. Cabral, N. Nishiyama, K. Kataoka, Acc. Chem. Res., 44 (2011) 999-1008.

[17] K. Kataoka, A. Harada, Y. Nagasaki, Adv. Drug Deliv. Rev., 47 (2001) 113-131.

[18] R. Gref, A. Domb, P. Quellec, T. Blunk, R.H. Muller, J.M. Verbavatz, R. Langer, Adv. Drug Deliv. Rev., 16 (1995) 215-233.

[19] Z. Ge, S. Liu, Chem. Soc. Rev., 42 (2013) 7289-7325.

[20] C. Deng, Y. Jiang, R. Cheng, F. Meng, Z. Zhong, Nano Today, 7 (2012) 467-480.

[21] J. Hu, G. Zhang, S. Liu, Chem. Soc. Rev., 41 (2012) 5933-5949.

[22] F. Meng, Z. Zhong, J. Feijen, Biomacromolecules, 10 (2009) 197-209.

[23] E.S. Gil, S.M. Hudson, Prog. Polym. Sci., 29 (2004) 1173-1222.

[24] S. Ganta, H. Devalapally, A. Shahiwala, M. Amiji, J. Control. Release, 126 (2008) 187-204.

[25] C.D.H. Alarcon, S. Pennadam, C. Alexander, Chem. Soc. Rev., 34 (2005) 276-285.

[26] M.A.C. Stuart, W.T.S. Huck, J. Genzer, M. Muller, C. Ober, M. Stamm, G.B. Sukhorukov, I. Szleifer, V.V. Tsukruk, M. Urban, F. Winnik, S. Zauscher, I. Luzinov, S. Minko, Nat. Mater., 9 (2010) 101-113. 
[27] H. Xu, W. Cao, X. Zhang, Acc. Chem. Res., 46 (2013) 1647-1658.

[28] M. Cap, L. Vachova, Z. Palkova, Oxid Med Cell Longev, DOI: 10.1155/2012/976753 (2012).

[29] N. Ma, Y. Li, H. Xu, Z. Wang, X. Zhang, J. Am. Chem. Soc., 132 (2010) 442-443.

[30] L. Wang, W. Cao, Y. Yi, H. Xu, Langmuir, 30 (2014) 5628-5636.

[31] P. Han, S. Li, W. Cao, Y. Li, Z. Sun, Z. Wang, H. Xu, J. Mater. Chem. B, 1 (2013) 740-743.

[32] H. Ren, Y. Wu, Y. Li, W. Cao, Z. Sun, H. Xu, X. Zhang, Small, 9 (2013) 3981-3986.

[33] T. Sun, Y. Jin, R. Qi, S. Peng, B. Fan, Macromol. Chem. Phys., 214 (2013) 2875-2881.

[34] T. Sun, Y. Jin, R. Qi, S. Peng, B. Fan, Polym. Chem., 4 (2013) 4017-4023.

[35] H. Ren, Z. Huang, H. Yang, H. Xu, X. Zhang, Chemphyschem, 16 (2015) 523-527.

[36] N. Ma, Y. Li, H. Ren, H. Xu, Z. Li, X. Zhang, Polym. Chem., 1 (2010) 1609-1614.

[37] P. Han, N. Ma, H. Ren, H. Xu, Z. Li, Z. Wang, X. Zhang, Langmuir, 26 (2010) 14414-14418.

[38] J. Thomas, Z. Dong, W. Dehaen, M. Smet, Macromol. Rapid Commun., 33 (2012) 2127-2132.

[39] Z. Dong, X. Li, K. Liang, S. Mao, X. Huang, B. Yang, J. Xu, G. Luo, J. Shen, J. Org. Chem., 72 (2006) 606-609.

[40] S. Mao, Z. Dong, J. Liu, X. Li, X. Liu, G. Luo, J. Shen, J. Am. Chem. Soc., 127 (2005) 11588-11589.

[41] X. Liu, L.A. Silks, C. Liu, M. Ollivault-Shiflett, X. Huang, J. Li, G. Luo, Y.-M. Hou, J. Liu, J. Shen, Angew. Chem., Int. Ed., 48 (2009) 2020-2023.

[42] F. Yu, P. Li, B. Wang, K. Han, J. Am. Chem. Soc., 135 (2013) 7674-7680.

[43] W. Cao, Y. Gu, T. Li, H. Xu, Chem. Commun., 51 (2015) 7069-7071.

[44] C. de Gracia Lux, S. Joshi-Barr, T. Nguyen, E. Mahmoud, E. Schopf, N. Fomina, A. Almutairi, J. Am. Chem. Soc., 134 (2012) 15758-15764.

[45] R. Fang, H. Xu, W. Cao, L. Yang, X. Zhang, Polym. Chem., 6 (2015) 2817-2821. 
[46] Y. Zhan, Z. Zhang, X. Pan, J. Zhu, N. Zhou, X. Zhu, J. Polym. Sci., Part A: Polym. Chem., 51 (2013) 1656-1663.

[47] F. Ma, J. Zhu, Z. Zhang, X. Pan, N. Zhou, X. Zhu, J. Polym. Sci., Part A: Polym. Chem., 51 (2013) 3159-3165.

[48] J. Zeng, Z. Zhang, J. Zhu, N. Zhou, Z. Cheng, X. Zhu, J. Polym. Sci., Part A: Polym. Chem., 51 (2013) 2606-2613.

[49] J. Zeng, J. Zhu, Z. Zhang, X. Pan, W. Zhang, Z. Cheng, X. Zhu, J. Polym. Sci., Part A: Polym. Chem., 50 (2012) 2211-2218.

[50] L.D. Zarzar, J. Aizenberg, Acc. Chem. Res., 47 (2014) 530-539.

[51] X.Y. Hu, T.X. Xiao, C. Lin, F.H. Huang, L.Y. Wang, Acc. Chem. Res., 47 (2014) 2041-2051.

[52] H. Ren, Y. Wu, N. Ma, H. Xu, X. Zhang, Soft Matter, 8 (2012) 1460-1466.

[53] X. Miao, W. Cao, W. Zheng, J. Wang, X. Zhang, J. Gao, C. Yang, D. Kong, H. Xu, L. Wang, Z. Yang, Angew. Chem., Int. Ed., 52 (2013) 7781-7785.

[54] Y. Yi, H. Xu, L. Wang, W. Cao, X. Zhang, Chem. Eur. J., 19 (2013) 9506-9510.

[55] X. Huang, R. Fang, D. Wang, J. Wang, H. Xu, Y. Wang, X. Zhang, Small, 11 (2015) 1537-1541.

[56] J. Tepper, L. Gunderson, Clinical Radiation Oncology, 3rd Edition ed., Elsevier health science2012.

[57] R.J. Passarella, D.E. Spratt, A.E. van der Ende, J.G. Phillips, H. Wu, V. Sathiyakumar, L. Zhou, D.E. Hallahan, E. Harth, R. Diaz, Cancer Res., 70 (2010) 4550-4559.

[58] Y. Goto, T. Kodaira, N. Fuwa, N. Mizoguchi, R. Nakahara, M. Nomura, N. Tomita, H. Tachibana, J. Radiat. Res., 54 (2012) 98-107.

[59] A.S. Hoffman, J. Control. Release, 132 (2008) 153-163.

[60] W. Cao, Y. Gu, M. Meineck, H. Xu, Chem. Asian J., 9 (2014) 48-57. 
[61] A.J. Swallow, Radiation Chemistry: An Introduction, Longman: London, U.K.1973.

[62] W. Cao, X. Zhang, X. Miao, Z. Yang, H. Xu, Angew. Chem., Int. Ed., 52 (2013) 6233-6237.

[63] N. Ma, H. Xu, L. An, J. Li, Z. Sun, X. Zhang, Langmuir, 27 (2011) 5874-5878.

[64] F. Meng, H. Xu, Y. Qi, K. Xu, X. Song, S. Niu, J. Li, Chem. Res. Chinese. U., 28 (2012) 319-322.

[65] J.J. Kresl, S.E. Schild, G.T. Henning, L.L. Gunderson, J. Donohue, H. Pitot, M.G. Haddock, D. Nagorney, Int. J. Radiat. Oncol., 52 (2002) 167-175.

[66] M.P. Bokoch, Y. Zou, S.G. Rasmussen, C.W. Liu, R. Nygaard, D.M. Rosenbaum, J.J. Fung, H.J. Choi, F.S. Thian, T.S. Kobilka, J.D. Puglisi, W.I. Weis, L. Pardo, R.S. Prosser, L. Mueller, B.K. Kobilka, Nature, 463 (2010) 108-112.

[67] D.M. Rosenbaum, S.G. Rasmussen, B.K. Kobilka, Nature, 459 (2009) 356-363.

[68] J.M. Dwyer, N. Engl. J. Med., 326 (1992) 107-116.

[69] W. Cao, Y. Li, Y. Yi, S. Ji, L. Zeng, Z. Sun, H. Xu, Chem. Sci., 3 (2012) 3403-3408.

[70] W. Cao, Y. Gu, M. Meineck, T. Li, H. Xu, J. Am. Chem. Soc., 136 (2014) 5132-5137.

[71] W. Cao, L. Wang, H. Xu, Chem. Commun., 51 (2015) 5520-5522.

[72] J. Nordberg, E.S.J. Arnér, Free Radical Biol. Med., 31 (2001) 1287-1312.

[73] D.J. Phillips, M.I. Gibson, Antioxid. Redox. Sign., 21 (2014) 786-803.

[74] S. Joshi-Barr, C. de Gracia Lux, E. Mahmoud, A. Almutairi, Antioxid. Redox. Sign., 21 (2014) 730-754.

[75] H. Xu, J. Gao, Y. Wang, Z. Wang, M. Smet, W. Dehaen, X. Zhang, Chem. Commun., (2006) 796-798.

[76] X. Zhang, H. Xu, Z. Dong, Y. Wang, J. Liu, J. Shen, J. Am. Chem. Soc., 126 (2004) 10556-10557.

[77] Y. Fu, J. Chen, H. Xu, C. Van Oosterwijck, X. Zhang, W. Dehaen, M. Smet, Macromol. Rapid Commun., 33 (2012) 798-804. 
[78] P.G. Wang, M. Xian, X. Tang, X. Wu, Z. Wen, T. Cai, A.J. Janczuk, Chem. Rev., 102 (2002) 1091-1134.

[79] A. de Mel, F. Murad, A.M. Seifalian, Chem. Rev., 111 (2011) 5742-5767.

[80] W. Cha, M.E. Meyerhoff, Langmuir, 22 (2006) 10830-10836.

[81] J. Yang, J.L. Welby, M.E. Meyerhoff, Langmuir, 24 (2008) 10265-10272.

[82] W. Cai, J. Wu, C. Xi, A.J. Ashe, M.E. Meyerhoff, Biomaterials, 32 (2011) 7774-7784.

[83] W. Cha, M.E. Meyerhoff, Biomaterials, 28 (2007) 19-27.

[84] W. Cha, M.R. Anderson, F. Zhang, M.E. Meyerhoff, Biosens. Bioelectron., 24 (2009) 2441-2446.

[85] L. Zeng, Y. Li, T. Li, W. Cao, Y. Yi, W. Geng, Z. Sun, H. Xu, Chem. Asian J., 9 (2014) 2295-2302.

[86] T. Li, Y. Yi, H. Xu, Acta Chim. Sinica, 72 (2014) 1079.

[87] L. He, H. Lai, T. Chen, Biomaterials, 51 (2015) 30-42.

[88] Y. Huang, L. He, W. Liu, C. Fan, W. Zheng, Y.-S. Wong, T. Chen, Biomaterials, 34 (2013) 7106-7116.

[89] R.C. Mulligan, Science, 260 (1993) 926-932.

[90] D. Fischer, Y.X. Li, B. Ahlemeyer, J. Krieglstein, T. Kissel, Biomaterials, 24 (2003) 1121-1131.

[91] G. Cheng, Y. He, L. Xie, Y. Nie, B. He, Z. Zhang, Z. Gu, Int. J. Nanomed., 7 (2012) 3991-4006.

[92] D. Yue, G. Cheng, Y. He, Y. Nie, Q. Jiang, X. Cai, Z. Gu, J. Mater. Chem. B, 2 (2014) 7210-7221.

[93] Y. He, Y. Nie, G. Cheng, L. Xie, Y. Shen, Z. Gu, Adv. Mater., 26 (2014) 1534-1540.

[94] W. Li, P. Zhang, K. Zheng, Q. Hu, Y. Wang, J. Mater. Chem. B, 1 (2013) 6418-6426.

[95] J. Liu, Y. Pang, J. Chen, P. Huang, W. Huang, X. Zhu, D. Yan, Biomaterials, 33 (2012) 7765-7774.

[96] J. Liu, Y. Pang, Z. Zhu, D. Wang, C. Li, W. Huang, X. Zhu, D. Yan, Biomacromolecules, 14 (2013)

1627-1636. 

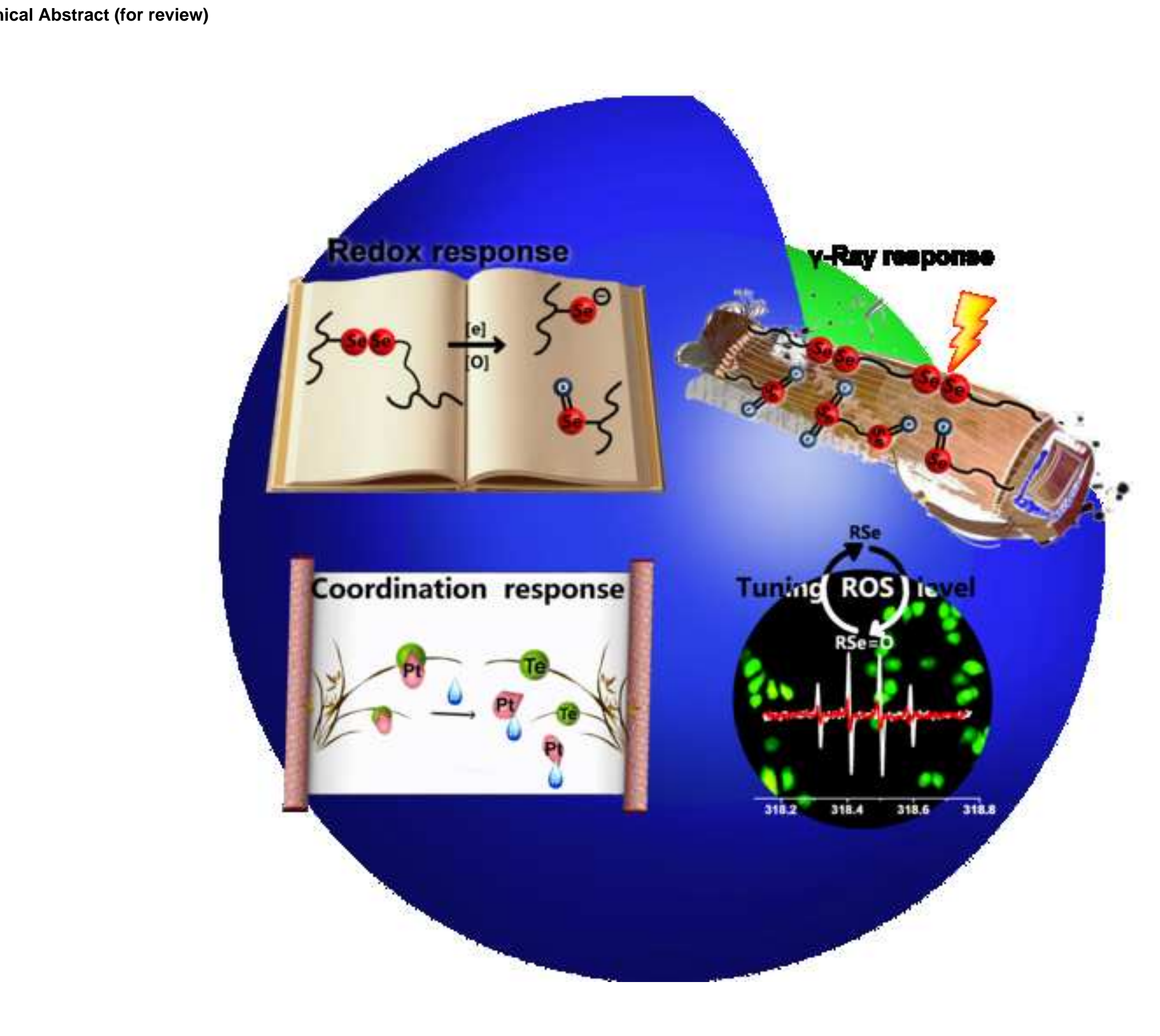

.
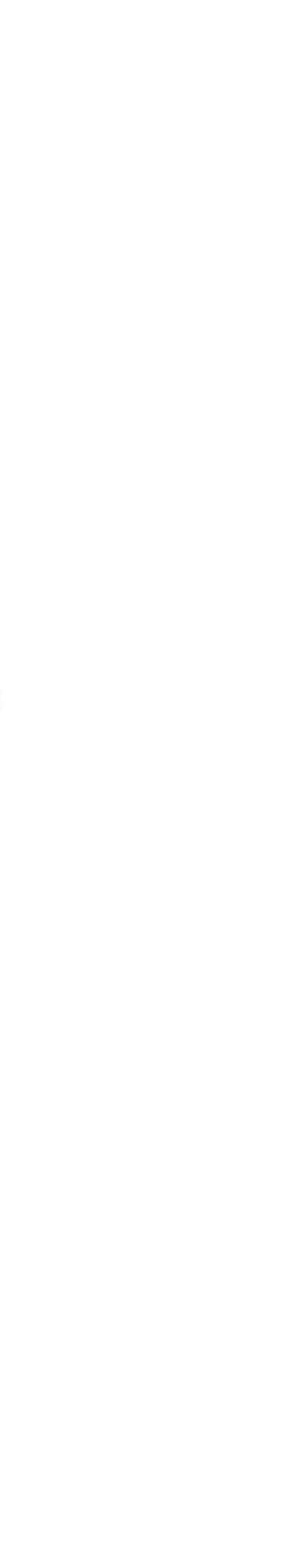